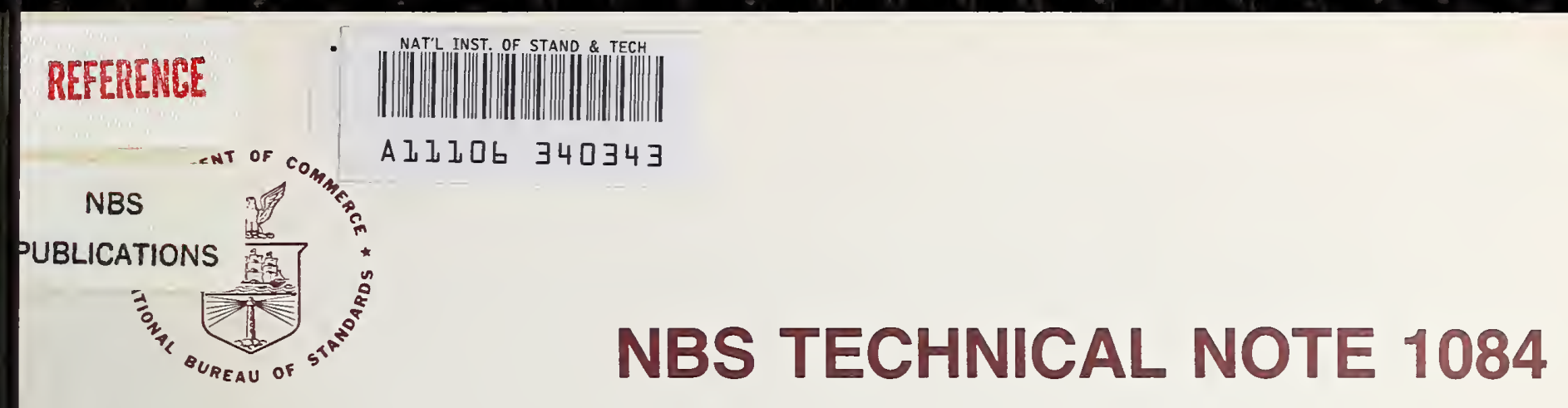

U.S. DEPARTMENT OF COMMERCE / National Bureau of Standards

\title{
Direct Measurement of the Electric Field of a Laser Pulse-Theory
}

Eric G. Johnson, Jr.

$Q C$

100

45753

No. 1084

1985 
he National Bureau of Standards' was established by an act of Congress on March 3, 1901. The Bureau's overall goal is to strengthen and advance the nation's science and technology and facilitate their effective application for public benefit. To this end, the Bureau conducts research and provides: (1) a basis for the nation's physical measurement system, (2) scientific and technological services for industry and government, (3) a technical basis for equity in trade, and (4) technical services to promote public safety. The Bureau's technical work is performed by the National Measurement Laboratory, the National Engineering Laboratory, the Institute for Computer Sciences and Technology, and the Center for Materials Science.

\section{The National Measurement Laboratory}

Provides the national system of physical and chemical measurement; coordinates the system with measurement systems of other nations and furnishes essential services leading to accurate and uniform physical and chemical measurement throughout the Nation's scientific community, industry, and commerce; provides advisory and research services to other Government agencies; conducts physical and çemical research; develops, produces, and distributes Standard Reference Materials; and provides calibration services. The Laboratory consists of the following centers:
- Basic Standards ${ }^{2}$

- Radiation Research

- Chemical Physics

- Analytical Chemistry

\section{The National Engineering Laboratory}

Provides technology and technical services to the public and private sectors to address national needs and to solve national problems; conducts research in engineering and applied science in support of these efforts; builds and maintains competence in the necessary disciplines required to carry out this research and technical service; develops engineering data and measurement capabilities; provides engineering measurement traceability services; develops test methods and proposes engineering standards and code changes; develops and proposes new engineering practices; and develops and improves mechanisms to transfer results of its research to the ultimate user. The Laboratory consists of the following centers:
- Applied Mathematics

- Electronics and Electrical Engineering ${ }^{2}$

- Manufacturing Engineering

- Building Technology

- Fire Research

- Chemical Engineering ${ }^{2}$

\section{The Institute for Computer Sciences and Technology}

Conducts research and provides scientific and technical services to aid Federal agencies in the selection, acquisition, application, and use of computer technology to improve effectiveness and economy in Government operations in accordance with Public Law 89-306 (40 U.S.C. 759), relevant Executive Orders, and other directives; carries out this mission by managing the Federal Information Processing Standards Program, developing Federal ADP standards guidelines, and managing Federal participation in ADP voluntary standardization activities; provides scientific and technological advisory services and assistance to Federal agencies; and provides the technical foundation for computer-related policies of the Federal Government. The Institute consists of the following centers:
- Programming Science and Technology

- Computer Systems Engineering

\section{The Center for Materials Science}

Conducts research and provides measurements, data, standards, reference materials, quantitative understanding and other technical information fundamental to the processing, structure, properties and performance of materials; addresses the scientific basis for new advanced materials technologies; plans research around cross-country scientific themes such as nondestructive evaluation and phase diagram development; oversees Bureau-wide technical programs in nuclear reactor radiation research and nondestructive evaluation; and broadly disseminates generic technical information resulting from its programs. The Center consists of the following Divisions:
- Inorganic Materials

- Fracture and Deformation ${ }^{3}$

- Polymers

- Metallurgy

- Reactor Radiation 


\section{Direct Measurement of the Electric Field of a Laser Pulse-Theory}

Eric G. Johnson, Jr.

Electromagnetic Technology Division

Center for Electronics and Electrical Engineering

National Engineering Laboratory

National Bureau of Standards

Boulder, Colorado 80303

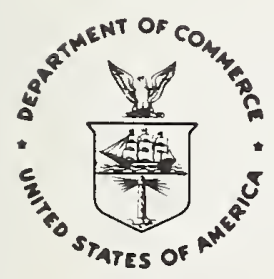

U.S. DEPARTMENT OF COMMERCE, Malcolm Baldrige, Secretary

NATIONAL BUREAU OF STANDARDS, Ernest Ambler, Director 
National Bureau of Standards Technical Note 1084

Natl. Bur. Stand. (U.S.), Tech Note 1084, 60 pages (Aug. 1985) CODEN:NBTNAE 


\section{CONTENTS}

Page

1. Introduction..................................... 1

2. Why know the Details of a Laser Pulse?....................... 4

3. Analytical Model Using Hermite Modes to Illustrate the

Basic Ideas of an Electric Field Measuring Apparatus........... 7

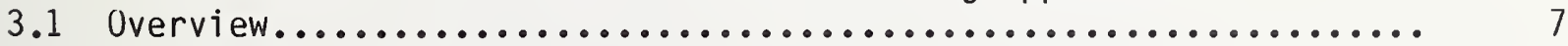

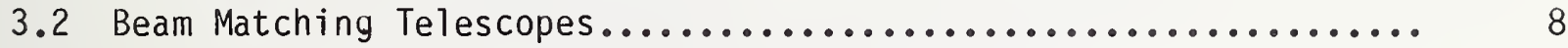

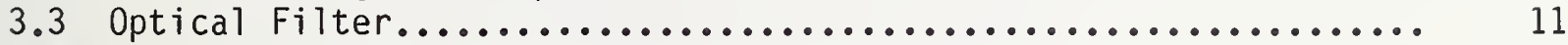

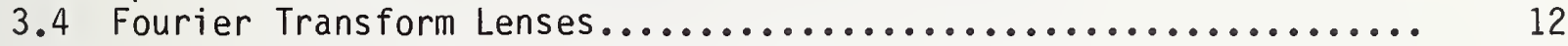

3.5 Fourier Plane--What Is Present There?........................ 14

3.6 Effects of Beam Clipping and Optical Axis Offset

between Fiber and Cross-Correlation Beam.................. 20

3.7 Design of a High Precision Positioning Device to Center

the Optical Axis of Each Fiber to the Optical Axis of

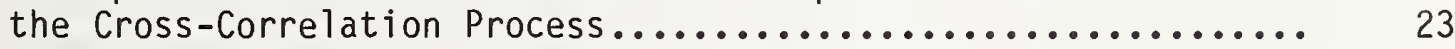

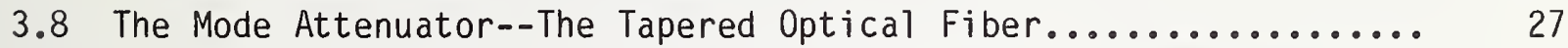

3.9 The Polarization Analysis Process--A Calcite Crystal........... 30

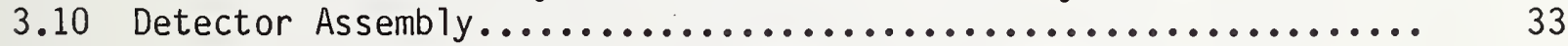

4. Realization of Nonlinear Filter Using Abstract Mathematics........ 34

4.1 Overview............................................ 34

4.2 Exact Equation Incorporating the Constraints of Fabrication...... 34

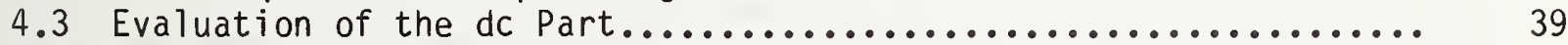

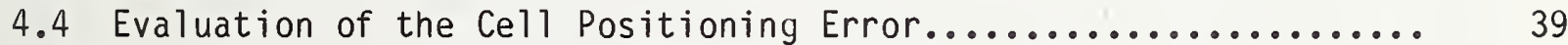

4.5 Evaluation of the Antisymmetric Nonlinear Part................ 40

4.6 Evaluation of the Antisymmetric Quasilinear Part.............. 43

4.7 Two Fabrication Strategies--Which One is Better?.............. 47

4.8 Necessary Work to Realize the Apparatus....................... 48

5. Conclusion........................................... 48

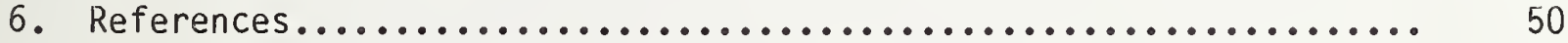



Direct Measurement of the Electric Field of a Laser Pulse--Theory

\author{
Eric G. Johnson, Jr. \\ Electromagnetic Technology Division \\ National Bureau of Standards \\ Boulder, Colorado 80303
}

We consider realizing an electric field measuring apparatus by using optical processing, tapered optical fibers, and a pair of detectors at the end of each optical fiber. Using an appropriate computer-generated optical filter, we show it is possible to discriminate among a set of orthonormal modes used to represent the spatial features of the electric field with a signal-to-noise ratio of at least 100 to 1 . If the positioning accuracies for various parts of the apparatus are properly set up, it is expected that the signal-to-noise ratio could be about 1000. The purpose of the tapered and graded-index fiber is to select the fundamental propagating mode in a fiber and to attenuate the other modes. The existence of this fiber allows the precise determination of the strength of each of the orthonormal modes being used as the spatial basis of the electric field before the optical processing. The detectors then measure the strength of each fundamental mode. There are 36 such modes for the apparatus under discussion--six for the $x$ dimension and six for the $y$ dimension. Propagation of the laser pulse is assumed to be along the $z$ axis. We use two detectors for each mode so that the polarization is determined.

This paper presents the conflicts in the design and gives a solution. The complete evaluation requires assembly of the proposed apparatus to assess final accuracy.

Key words: beam profile measurements; cross-correlation; electric field measurement; graded-index fibers; laser pulses; matched filters; mode analysis; mode filtering; numerical analysis; optical fibers; optical filter; singular value decomposition; total beam control

\title{
1. Introduction
}

We at NBS [1-4] have been developing an electric field measuring system to measure the time and spatial features of a laser pulse with at least 1.0 percent accuracy. If certain critical elements can be set up, we expect the 
accuracy to be 0.1 percent. We have found a number of conflicting constraints on the design of the equipment. We address these constraints in this paper. First, we show why the optical processing is desirable for proper electric field measurements. Second, using simplified analytical models, we illustrate the technical conflicts that result from using optical processing. They are: (1) mode mixing due to clipping, various offsets in the optical axis, and various mode mismatches at several locations; (2) mode mixing due to the Fourier transform process; and (3) attenuating the undesired modes which result from the cross-correlation process. From these illustrations and the development of the computer-generated filter, which has nonlinear features, we show how to get a resolution of the conflicts.

We yet this resolution in three key technical stages:

1. The first stage uses a computer-generated spatial filter to act as a laser-basis mode to fundamental optical-fiber mode converter. In this way we get a decomposition of the electric field of a laser pulse by an orthonormal mode analysis of the original laser pulse. Because there are fundamental constraints in the allowed form of the electric field at the Fourier plane resulting from the matched filter cross-correlation process, it is necessary to attentuate the resulting undesired electric field signals at the selected spatial locations in this Fourier plane.

2. The second stage uses a graded-index optical fiber at each of the selected spatial locations in the Fourier plane. Each optical fiber starts with $300 \mu \mathrm{m}$ core diameter size and is adiabatically pinched to a single-mode core diameter around $10 \mu \mathrm{m}$. After the pinch, these fiber cores return to the $300 \mu \mathrm{m}$ diameter. This stage attenuates a 11 but the fundamental propagating modes in the optical fiber.

3. The third stage has a detector which measures the power from each fiber. This step is done if there is no need for measuring the polarization or the carrier phase of the laser pulse. If there is a need for the latter, then it is necessary to run the pulse from the optical fiber though a polarization separation device and finally to heterodyne at the detectors the resulting signals with a selected reference beam from the same laser pulse. 
The paper discusses the necessary equipment stages to make a direct measurement of the electric field in the original laser pulse. These are: (1) a beamwidth, phase front, and an optical axis matching telescope; (2) the optical filter with beam steering; (3) a pair of lenses to perform the Fourier transform and to allow precision adjustment of the focal length; (4) four 3by-3 assemblies of the fiber optic units with appropriate six degree of freedom adjustments; (5) the necessary elements for polarization assessment; and finally (6) four 6-by-3 detector arrays with associated electronics for recording the signals of interest. We are assuming that six orthonormal modes for each dimension are sufficient. The critical parts in this apparatus are: (1) the optical processing filter, (2) the positioning device for the individual optical fibers, and (3) the tapering process of the individual fibers. Less critical parts are: (1) the detector assembly, (2) the mode matching telescope, and (3) the Fourier transform lens assembly. The first needs proof of final accuracy and the last just needs to be done in the context of the entire apparatus.

The paper is structured at two mathematical levels. The first level uses an analytical formulation of the problem with Hermite polynomials as the orthonormal basis modes. The second level shows the more complex mathematical steps which require a digital computer to generate the required optical filter. In this way all the technical issues for construction of the electric field measuring apparatus should be clear.

Section 2 discusses why knowledge of the electric field is eventually necessary for complete measurement of a laser pulse. Also this section gives a brief functional diagram of the desired measuring unit so the reader has a visual sense of how the apparatus would work.

Section 3 addresses the basic technical ideas in developing an electric field measuring apparatus. Here we use Hermite modes so we can have analytical formulas to illustrate the ideas. This section contains the main body of ideas about the electric field measuring apparatus. Each idea is discussed to the detail necessary for the reader to understand what is so. There is a collection of figures which illustrate the basic physical construction ideas at each stage. The critical dimensions are indicated in the text. The actual chosen values are suggested so that the reader will have a concrete idea of 
what should work. The final test will require construction of the unit. Discussion of that final test will be in a second paper after the unit is built and tested. The purpose of this paper is to present the ideas so that such an apparatus could be built when it is economically desirable. The key ideas are: (1) the beam matching by a pair of telescopes [5], (2) the crosscorrelation process by an appropriately constructed optical filter [6], (3) the Fourier transform lens, which has a variable focal length, (4) the tapered fiber optic assembly which causes attenuation of all modes of propagation except the fundamental mode [7-10], (5) estimating and design for minimizing the impact of various alignment and mode matching steps in the apparatus [1112], and in addition, (6) devising the necessary polarization [13] and detection techniques [14-17] for the resulting fundamental mode.

Section 4 addresses the problems in construction of the computergenerated filter [18]. Here we introduce the complex mathematics [19] necessary to get a filter which will allow optical processing with high precision.

Section 5 indicates what advances in construction techniques are necessary to physically realize this measurement apparatus.

2. Why know the Details of a Laser Pulse?

The complete specification of how a laser pulse evolves in space requires critical details of the electric field such as the electric field profile transverse to the direction of propagation over the time period of the pulse. Such measurements are not easy and, in fact, many would say impossible. With current laser sources, mechanical adjustment parts, optical fibers, and electrochemical deposition techniques, we are beginning to get to the technical capability where it is possible to measure the electric field of a laser pulse. This paper describes how this can be done, the technical problems in realizing that goal, and the degree the goal can be realized.

Why would anyone want to know the details of a laser pulse? The primary reason is that we can then have better beam control of the spatial profile, of the time dependence of the pulse, of the time and spatial dependence of the polarization, and of the deviations from the carrier frequency. Better beam control means we can do more precise operations with these laser pulses. 
To illustrate, laser pulses are now being used as more and more precise means for delivering energy of a specific form to a surface of a material to modify that material in various ways. Examples range from simple welding or heat treatment, to the more esoteric uses such as chemical phase changes at localized locations (read and write cycles of optical disks). In these illustrations, it becomes more and more appropriate to have a means to measure the electric field of a laser pulse. The final applications may not need total beam control. Ultimately there needs to be a precise measurement means to verify the appropriate level of beam control in the various applications of these laser pulses. Some new areas of technical endeavor where we suggest that total beam control will be valuable are: (1) in the area of parallel processing for pattern recognition of two-dimensional processes (for example, time dependent checking of the heat distribution in a computer chip), (2) in the area where a chemical reaction is taking place on a surface (here the laser's time and spatial pattern may need to be specially controlled to drive efficiently the chemical reaction in conjunction with the surface catalyst), and (3) in the area of biological development in cells where it would be helpful to selectively cause a disturbance to the biological process with a specific pattern and with a specific pulse shape.

Current means for measuring the details of laser pulses are incomplete. They can be designed to measure the flux (irradiance) $\left(\mathrm{W} / \mathrm{m}^{2}\right)$ or fluence $\left(\mathrm{J} / \mathrm{m}^{2}\right)$ with a time resolution ranging from picoseconds to seconds. The details of the phase front are usually only known by inference. This means that we have lost at least half of the necessary information. Examples of such measurement devices are: (1) single detectors which scan the laser beam and (2) twodimensional detector arrays [20-21].

Figure 1 shows a block diagram of an electric field measuring apparatus. The numbers in this figure are references to later figures which show the details of that block. Briefly, the steps in making an electric field measurement are:

Step A: get a sample of the laser pulse of interest by using a beamsplitter or a high spatial frequency grating. 


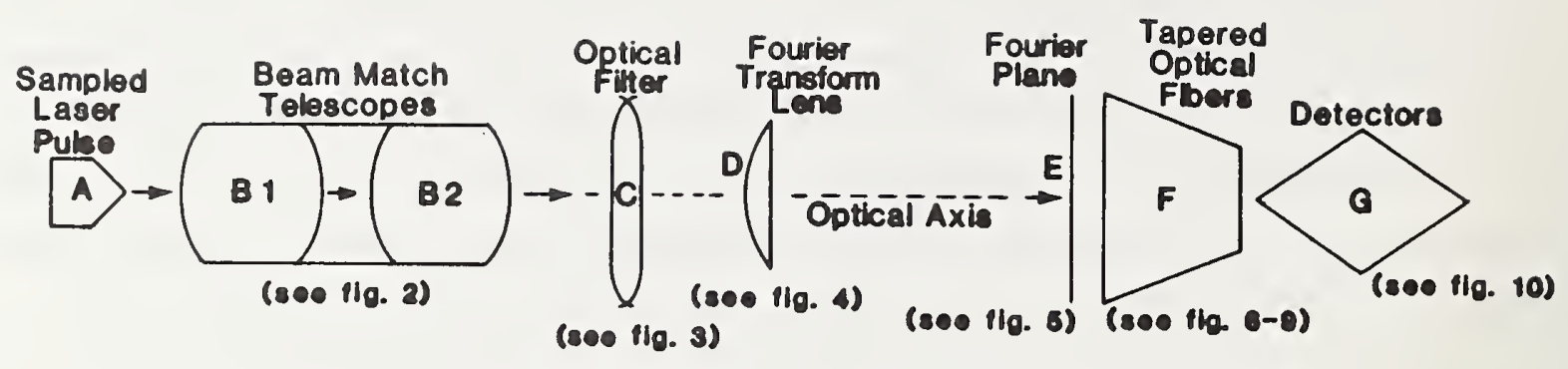

Figure 1. Block diagram of the steps in the electric field measuring apparatus.

Step B: modify this pulse so that it has a beamwidth, a phase front, and an optical axis that are matched to an optical filter. Obviously this matching process will require high quality laser sources and high mechanical stability in the beam sampling and beam modification. The telescope B1 and the reverse telescope B2 with appropriate mechanical adjustments accomplish this function.

Step C: use an optical filter which is coded with the following features, namely (1) unique beam steering for each orthonormal spatial decomposition of the electric field and (2) a proper intensity weighting for the orthonormal decomposition so that there is a cross-correlation pattern which has a nearly Gaussian profile of standard width for the desired correlation and that is a linear combination of the higher order Hermite modes for the undesired correlations.

Step D: use an adjustable Fourier transform lens to cause the resulting pattern exiting from the optical filter to form the cross-correlation patterns at the Fourier transform plane of this lens.

Step E: mount four blocks of 3-by-3 arrays of optical fibers. Each fiber is located so that its optical axis is centered on the Gaussian profile resulting from the desired cross-correlation process. The fundamental mode of each graded-index fiber is Gaussian-like and hence is to be matched in phase front, in beamwidth, and in beam center to the Gaussian profile resulting from the cross correlation. To account for various errors in fabrication of these blocks, they are mounted on positioning equipment which will allow 0.1 um 
positioning accuracy in all six degrees of freedom. The final errors in fabrication may be reduced by proper use of the beam bending design in the optical filter and by using piezoelectric units to center the optical axis of each optical fiber relative to the optical axis of the desired crosscorrelation mode.

Step F: each optical fiber has been modified so that its optical core changes slowly from 300 um diameter to 10 um diameter and back over a distance of $3 \mathrm{~cm}$. These optical fibers are immersed individually in an index matching fluid so that we can get efficient attenuation of the modes not being sustained by the restricted core diameter. Each optical fiber is polished at the front and back so that there is accurate mode coupling at the beginning and a good match to a calcite crystal at the end. The latter results in the separation of the two polarization states of that propagating single mode in the optical fiber. The thickness of the crystal is long enough to allow separation of the polarization states and yet is short enough to minimize the beam spreading of the fundamental mode in the crystal.

Step G: A detector is situated to measure the power in each polarized mode. To allow heterodyne action, it is desirable to structure the detectors so that there is some form of local oscillator signal with a carrier frequency near that of the laser pulse. This step requires some form of integrated optics to be associated with each detector as well as some form of integrated electronics to record the time shape of the laser pulse. We do not discuss how this is done.

3. Analytical Model Using Hermite Modes to Illustrate the Basic Ideas of an Electric Field Measuring Apparatus

3.1 Overview

In this section, there are nine subsections. Each subsection deals with some aspect of processing the critical electric field information in the laser pulse. Subsection 3.2 addresses the two beam matching telescopes. Subsection 3.3 addresses the optical filter from a construction point of view. Subsection 3.4 addresses how the Fourier transform lenses operate. Subsection 3.5 addresses the mechanical positioning of the optical fiber units and what is 
present at the Fourier transform plane for the simplified analytical case. Subsection 3.6 addresses the errors in matching the optical axis of the graded-index fiber to the optical axis of a particular cross correlation processor. Also it addresses what the finite size of the optical fiber does to the information. At this stage it is appropriate to use these same formulas to estimate the effects of the finite size apertures at other stages of this apparatus. Subsection 3.7 shows how to construct the positioning equipment to minimize the positioning errors. Subsection 3.8 addresses how the mode attenuation process works within the tapered fiber. Subsection 3.9 addresses the polarization separation process. Finally we close this section with the brief description in subsection 3.10 of the detector process.

\subsection{The Beam Matching Telescopes}

Figure 2 shows the configuration of the four lenses used to set the laser pulse to a standard beamwidth and to a flat phase front. There are two telescopes. One acts to create a local image and a flat phase front of the incident laser pulse. The second telescope acts as a beam expander to create a standard beamwidth and a flat phase front at the optical filter location indicated as $q_{10}$ in figure 2. The $f_{n}$ are the focal lengths of the simple lenses. The $f_{2}$ and $f_{8}$ are $2000 \mathrm{~mm}$ planoconvex lenses. The $f_{4}$ and $f_{6}$ are $-2000 \mathrm{~mm}$ planoconcave lenses. The distance between each critical optical stage is indicated by $d_{n}$. The critical optical stages are indicated by the complex beam parameters for an ideal Gaussian beam. Thus we can use $a_{n}=i b_{n}+z_{n}$, where $b_{n}=w_{n}{ }^{2}(\pi / \lambda), w_{n}$ is the beamwidth at the nth location, and $-z_{n}$ is the relative distance to a point where the Gaussian beam has the width $w_{n}[3,5]$. We use the Gaussian beam as a means for design choice. The actual laser beam need not be Gaussian. The final adjustment procedures would use the entire apparatus to select the required standard beamwidth and the flat phase front. How that is done will be described in the conclusions. For now we explain how to do the crude adjustments of the beam matching telescopes.

There are two relationships which allow determination of position and status of the laser beam at each optical stage.

$$
\text { Type 1: } q_{n+1}=q_{n}+d_{n} \text { for a simple displacement with } f_{n}=0 \text {. }
$$



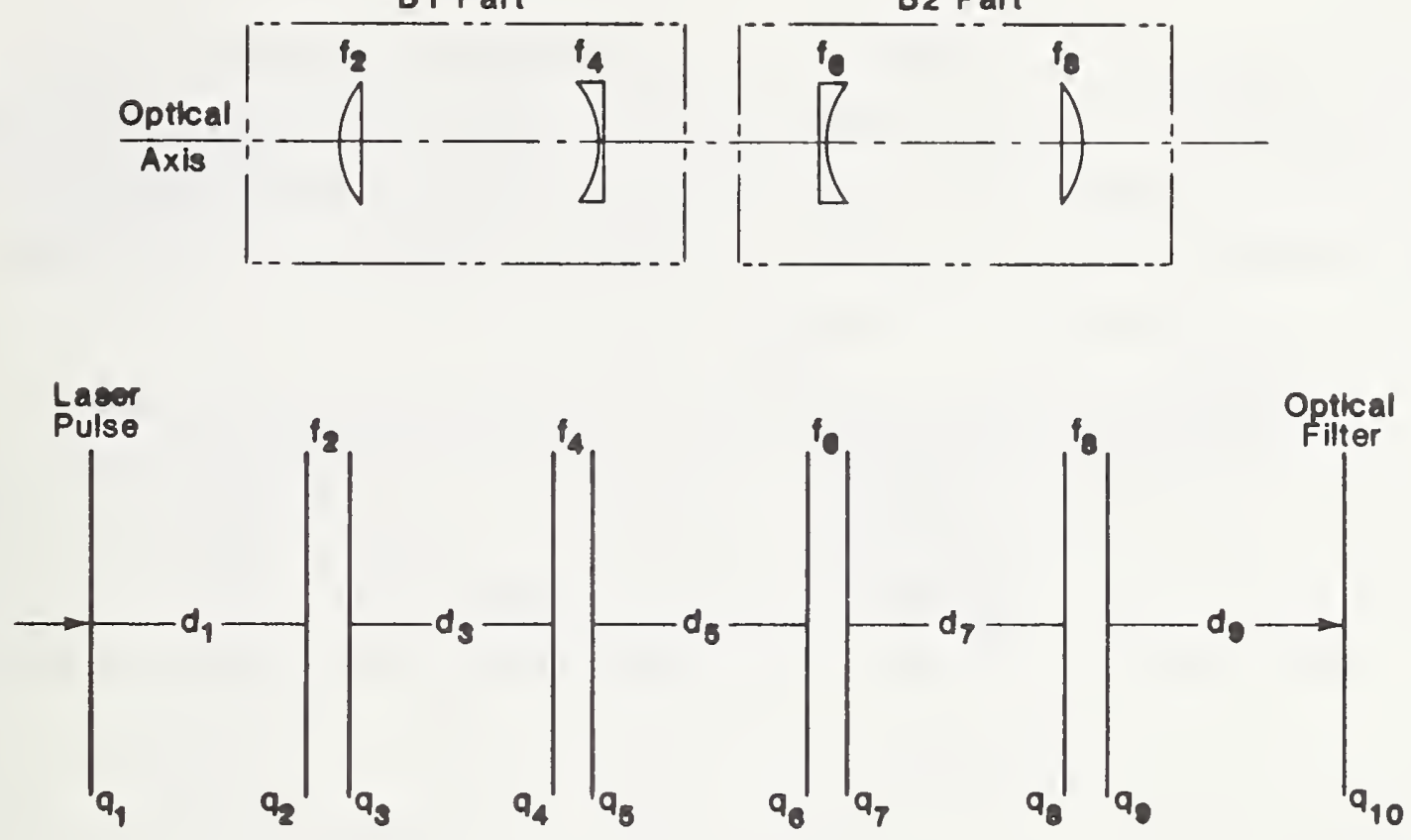

Figure 2. Beam matching telescope, step $B$ in figure 1.

Type 2: $q_{n+1}=-f_{n}+f_{n} 2 /\left(f_{n}-q_{n}\right)$

for the action of the thin lens with $d_{n}=0$.

In a calculation sequence, we would assume that $f_{n}, b_{1}, z_{1}, b_{10}$, and $z_{10}$ are specified. We fix the $d_{1}$ and $d_{9}=100 \mathrm{~mm}$ for the design conditions. We use the constraint parameter $T=\left(d_{7} / d_{3}\right)^{1 / 2}=1200 \mathrm{~mm}$ as a means for setting a convenient dynamic range for $d_{7}$ and $d_{3}$ of about $800 \mathrm{~mm}$. We do the computation by a self consistency sequence. First we compute the value of $b_{5}$ using the current values starting from optical stage $q_{1}$. Next we compute the value of $b_{5}$ using the current values starting from the optical stage $q_{10}$ and going backwards through the stages. The sequence is iterated until the computed $b_{5}$ in both directions are equal within the accuracy desired. For this iteration process to work, we need to specify $d_{3}$ and $d_{7}$ for each iteration. We constrain $d_{7}=T^{2} / d_{3}$ at each iteration. We get a new $d_{3} N$ from the old $d_{3}{ }^{0}$ by the relation

$$
d_{3 N}=d_{3} 0\left[b_{5} / b_{6}\right]^{1 / 4} \text {. }
$$


To start the iteration process we use the condition $d_{3}=T /(M)^{1 / 2}$, where $M=\left(b_{1} / b_{10}\right)^{1 / 2}$. This $M$ is a measure of the magnification change necessary to match the input beamwidth and phase front at 1 to the standard output beamwidth and phase front at 10 where we assume $w_{10}=10 \mathrm{~mm}$ and a $\mathrm{flat}$ phase front.

The remaining technical points about the beam matching telescope are: aberrations, extraneous light due to reflections and the positioning equipment necessary for the above adjustments, and to fix the optical axis. We address briefly these points. The aberrations are expected to be small for the choice of $F$ number and field of view. If it is desirable to make them even smaller, then it is desirable to either use two lenses in place of each lens to make the adjustment for minimum aberration over a selected range of magnifications and phase fronts. In the construction of the prototype apparatus, we suggest that this correction be ignored. Reflections from the surfaces can be dealt with two ways, namely use an antireflection coating on each lens for the wavelength range of interest or use off-axis mirrors. Again for the first apparatus, we suggest transmission optics with antireflection coating set for $1.0 \mu \mathrm{m}$. There remains one final point, the selection of positioning equipment for these lenses. We suggest that an optical rail with a means to position each lens on the rail to an accuracy of $1 \mu \mathrm{m}$ is necessary. Each lens unit would have a means to assure that its optical axis matches the reference optical axis with an accuracy of $1 \mu \mathrm{m}$. Because the total distance from stage $q_{1}$ to stage $q_{10}$ will change for a generic laser pulse, it is desirable to design a means to eliminate the influence of this total distance change on the rest of the apparatus. One way to do this is to use a pair of $45^{\circ}$ prisms with antireflecting coating on the surfaces where the laser pulse enters and exits. Under these circumstances the change in distance is perpendicular to the rest of the apparatus and would not affect their relative locations. As always there is a price, it is necessary to correct for the change in the phase front and polarization state of the laser pulse due to these beam turning operations which can be done with appropriate use of Hermite modes. This completes the discussion of this subsection except to remind the reader that there is a need to specify the aperture size of each 1ens. One criterion for selection of the aperture will be discussed in section 3.6 . 


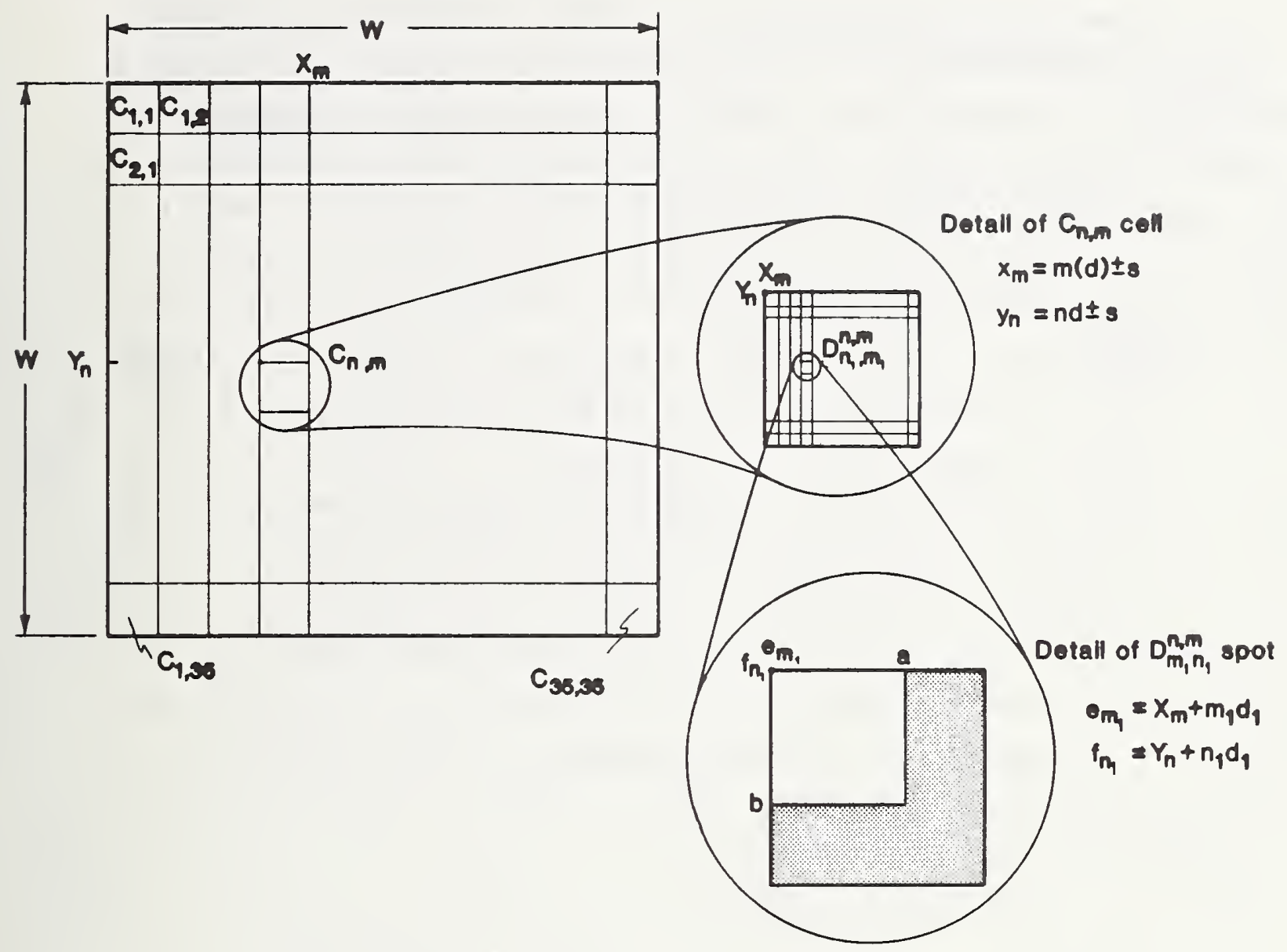

Figure 3. Optical filter, step C in figure 1 (front view).

\subsection{Optical Filter}

In this section we address the physical construction of the optical filter. The particular construction parameters are specific to what can be fabricated at one particular facility. If a different optical-filter-generation facility is used then the construction parameters may be different. Figure 3 shows the sequence. First there is a glass plate with $W=70 \mathrm{~mm}$. Each cell indicated by $c_{n, m}$ has $d=10 \mu m$ with the range of $n$ and $m$ from 1 to 35 . The positioning accuracy of each cell has $s=0.15 \mu \mathrm{m}$. Within each cell are 400 spots with their corner locations at the coordinates $e_{m_{1}}, f_{n_{1}}$. Each spot $D$ has a size given by an $a$ and $b$ which are uniquely specified for each cell. This implies that 98.0 million data values must be specified for a given optical filter. 
We assume that the radiation is 100 percent transmitted through the $D^{n, m_{n 1}}{ }_{n 1}$ spot where the area is clear and 0 percent transmitted through the shaded area of that same spot. This cell simply samples the electric field illuminating the filter and passes that part within each shaded region. This fact means there is no true gray scale for the optical filter. There is some sampling noise due to this binary sampling process and thus a restriction in dynamic range of the action of the filter on the laser pulse. Beyond these constraints, there is a second form of sampling error because the allowed values of a are in discrete steps of $0.3 \mu \mathrm{m}$ and because the allowed values of $b$ are in discrete steps of $0.25 \mu \mathrm{m}$. The range of a is from $2 \mu \mathrm{m}$ to $8 \mu \mathrm{m}$. The range of $b$ is 0 to $10 \mu \mathrm{m}$. These allowed conditions are unique to the optical fabrication facility. Other facilities would change these conditions. Section 4 will address the consequences of these sampling constraints. We assume for simplicity in this section that we have a gray scale filter with no restrictions on dynamical range. By this step we can use analytical expressions for the discussion. It will be necessary to mount the filter perpendicular to the optical axis and to make sure there is no rotation of this filter relative to the axis.

\subsection{Fourier Transform Lenses}

Figure 4 shows the nominal structure of the pair of lenses used to define the Fourier transform segment of this apparatus. The anodized tube is used to reduce the spurious radiation on the optical fibers in the Fourier plane. This tube is only representational. The proper shielding may be different. We use the same design formulas as were used in subsection 3.2. Here we use two lenses and we know the beam parameters at $q_{1}$ to be $w_{1}=10.0 \mathrm{~mm}$ and the phase front is flat. A critical adjustment of these two lenses will make the effective focal length at stage $q_{6}$ show that $\lambda f_{\text {eff }}=1 \mathrm{~mm}^{2}$ is true for the wavelength of interest. For our discussion we assume $\lambda=1.00 \mu \mathrm{m}$. Incidentally, this wavelength change will be important in the adjustment of the beammatch stage just as it is important in this Fourier transform stage. The design conditions are to have $q_{6}$ with a beamwidth $w_{6}=(\lambda / \pi) \mathrm{feff}_{\mathrm{f}} / \mathrm{w}_{1}$ and $z_{6}=0$. We assume for this discussion that the two lenses are plano-convex with $f_{2}=2200 \mathrm{~mm}$ and $f_{4}=1800 \mathrm{~mm}$. The distance $d_{1}$ is controlled by the 

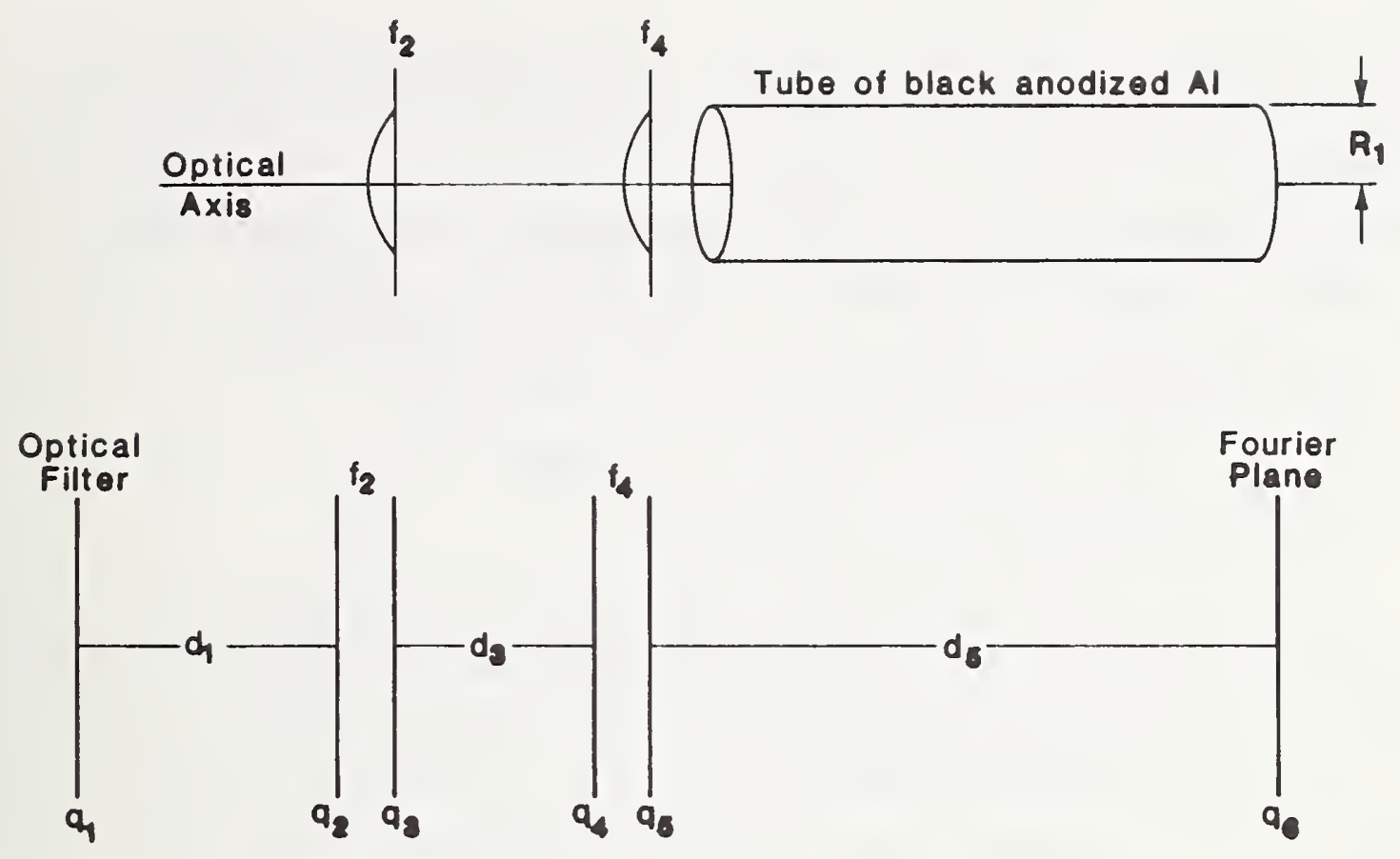

Figure 4. Fourier transform lenses, step $D$ in figure 1.

mounting hardware for the filter, by the thickness of the lenses, and by the changes in $d_{3}$ and $d_{5}$. We assume $d_{1}$ is about $100 \mathrm{~mm}$. The distance $d_{5}=982 \mathrm{~mm}$ and is set by requiring $f_{\text {eff }}=1000 \mathrm{~mm}$. The distance $d_{3}$ is adjusted to satisfy the constraints at $q_{6}$. The key approximate formulas are:

$$
f_{\text {eff }}=f_{2}\left(f_{4}-d_{5}\right) / f_{4},
$$

and

$$
d_{3}=d_{a}-f_{2}^{2}\left(f_{2}-d_{1}\right) /\left(b_{1}\right)^{2} \text {. }
$$

with the definition

$$
d_{a}=f_{2}+f_{4}-f_{4} 2 /\left(f_{4}-d_{5}\right) .
$$

These are derived by noting the size of $b_{6}$ and $b_{1}$ relative to $f_{2}$ and $f_{4}$ in the exact formulas. We get $d_{3}=39 \mathrm{~mm}$ for our example. There needs to be a precision adjustment of $1 \mu \mathrm{m}$ accuracy for stages $q_{2}$ and $q_{4^{*}}$. The value of $d_{1}$ will change because the distance between $q_{1}$ and $q_{6}$ is fixed. The distances chanye 
with wavelength to keep $\lambda f$ eff constant. This constant keeps the Fourier transform plane unchanged relative to the optical filter. We first use the approximate equations to set starting values and then use a self-consistency iteration procedure with the exact equations to finally get the correct $d_{3}$ and $d_{5}$ values. $b_{1}$ and $b_{6}$ are constant in this iteration process.

Again precision adjustment equipment is necessary.

\subsection{Fourier Plane--What Is Present There?}

The Fourier plane contains the results of the cross-correlation from the optical filter and the Fourier transform lenses acting on the laser pulse. Figure 5 shows four physical structures in this plane. Each structure is held by a three-axis positioner with accuracy of $0.1 \mu m$ in each dimension, a single rotation unit of accuracy 1 mrad, and by two double tilt positioners with accuracy of $1 \mathrm{mrad}$. Each structure is made of a 3-by-3 array of optical fibers mounted in a square pattern indicated by the dimensions in figure 5 . The approximate dimensions of the structure set $d_{1}=20 \mathrm{~mm}$. The hole diameter is $d_{5}=350 \mu \mathrm{m}$, where it is assumed that each optical fiber has a diameter $d_{6}=325 \mu \mathrm{m}$ including the protective sheath and an graded-index core diameter of $300 \mathrm{~mm}$. The actual placement of the holes in the electroform sheet, as indicated by the distance $d_{4}$, can be either periodic for each 3-by-3 array or uniquely located for each structure. If there are no significant ghosts due to the cross-correlation process in the computer generated filter, then a periodic structure is adequate. If there are significant ghosts, then a nonperiodic structure is necessary. In our discussion we assume that the periodic structure is adequate. This point will be further discussed in section 4. The remaining dimension is the absolute positioning of the four structures as indicated by $D_{2}$ and $D_{3}$. Using coordinate notation $\left(D_{2}, D_{3}\right)$, we have four number pairs with the values in millimeters each of $(6.28,6.28)$, $(6.28,-8.7),(-8.7,6.28)$, and $(-8.7,-8.7)$. These coordinates locate where the corner optical fiber nearest the optical axis has its center relative to that optical axis. There is a correction to these coordinates to get the $d_{2}$ and $d_{3}$ shown in figure 5 . This depends on the final construction details of the 3-by-3 structures. That correction is expected to be about $5 \mathrm{~mm}$. The final dimension of the spacing between the optical fibers has $d_{4}=5 \mathrm{~mm}$ for the periodic case. 


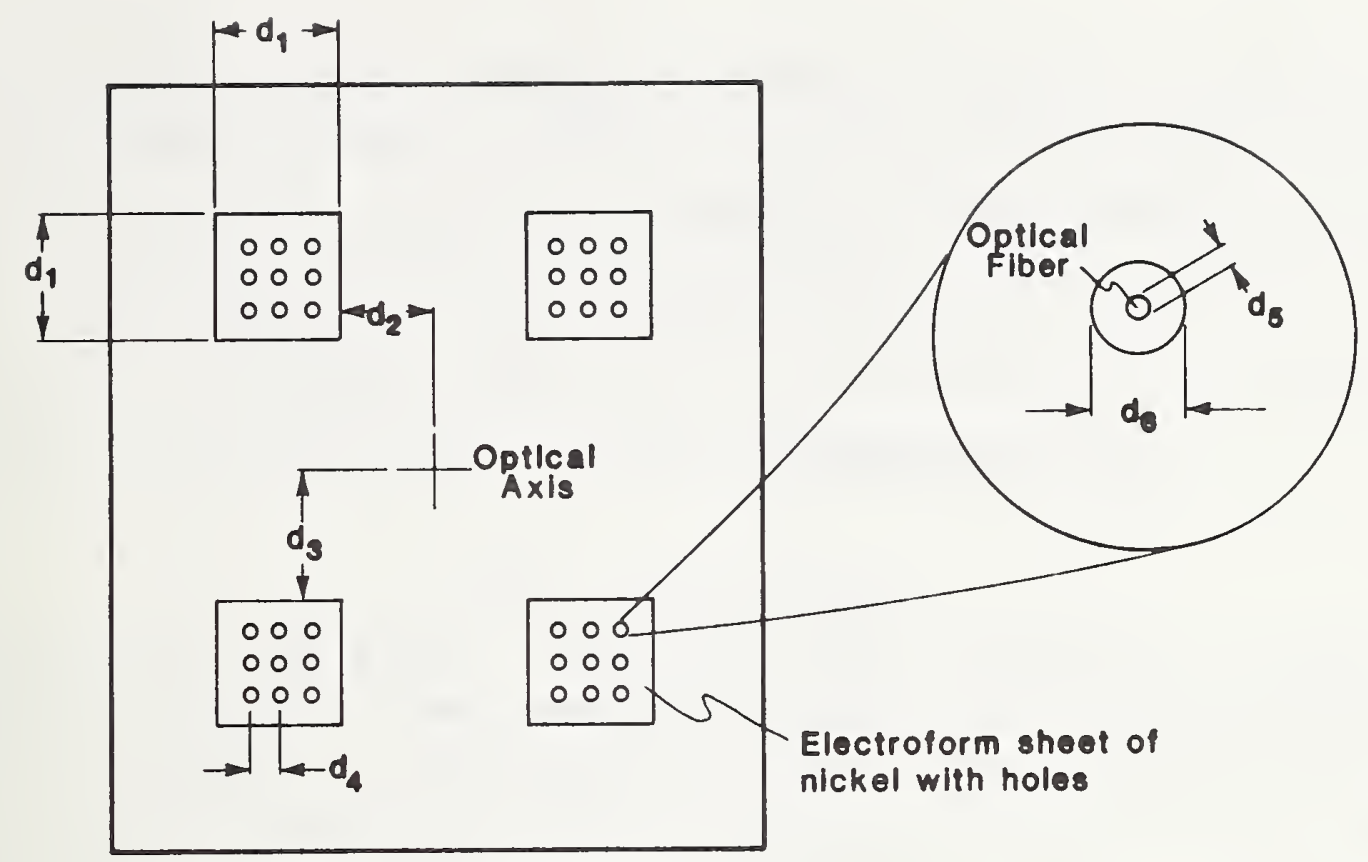

Figure 5. Fourier plane, step $E$ in figure 1 (front view).

The physical dimensions are now defined, and we are ready to look at the analytical formulas to show the kind of cross-correlation pattern produced in the Fourier plane.

We represent the electric field just before the optical filter by the form in eq (7)

$$
E_{\ell}(x, y)=\sum_{n, m=0}^{\infty} A_{n, m, \ell} H_{n}(\sqrt{2} x / w) H_{m}(\sqrt{2} y / w) W(x) W(y) /\left(C_{n} C_{m}\right)
$$

where the following definitions are noted:

$$
\begin{aligned}
& H_{n}(\sqrt{2} x / w)=\left.\left(\frac{d}{d z}\right)^{n} \exp \left[-z^{2}+2 \sqrt{2} x z / w\right]\right|_{z=0}, \\
& W(x)=\exp \left[-(x / w)^{2}\right], \\
& \left.Z\left(x, z_{1}, w\right) \equiv \exp \left[-z_{1}^{2}+2 x z_{1} \sqrt{2} / w\right] W(x)\right|_{z_{1}=0}, \\
& C_{n}=2^{n} n ! / \sqrt{\pi},
\end{aligned}
$$




$$
\begin{aligned}
& c_{n} \delta_{n_{1}}^{n}=\frac{\sqrt{2}}{w} \int_{-\infty}^{\infty} d x H_{n}(\sqrt{2} x / w) H_{n_{1}}(\sqrt{2} x / w)[w(x)]^{2}, \\
& H_{n}(-\sqrt{2} x / w)=(-1)^{n} H_{n}(\sqrt{2} x / w),
\end{aligned}
$$

and

$$
\ell=x \text { or } y \text { polarizations. }
$$

Here the $w$ is the Gaussian width of the beam. \& represents the two polarization states and the $n$ and $m$ represent the mode label for $x$ and $y$ coordinates of Hermite functions. $C_{n}$ and $C_{m}$ are the necessary normalizations for the orthonormal expansions. $A_{n, m, l}$ are the strengths of each mode in the electric field. $Z$ represents the generator of the Hermitian modes. $z_{1}$ is set zero after the differentation process. The symmetries of the Hermitian modes are also indicated.

We assume that the optical filter can be represented as

$$
T(x, y)=T(x) T(y)
$$

This means we can separate the two dimensions and do the analysis with one dimension only. With this assumption we can write the electric field after the filter as

$$
E_{\ell}(x, y)=\frac{w^{2}}{2} \sum_{n, m=0}^{\infty} A_{n, m, \ell} E_{n}(x) E_{m}(y) /\left(C_{n} C_{n}\right),
$$

where we have the one-dimensional field mode as

$$
E_{n}(x)=\left(\frac{d}{d z_{1}}\right)^{n} Z\left(x, z_{1}, w\right) T(x)
$$

The definition of the filter function is

$$
\left.T(x)=\sum_{j=0}^{5} B_{j} H_{j}(\sqrt{2} x / w) \cos [u(j) x+v(j)] W(x) / W 1(x) C_{j}\right) .
$$


Here we require the filter function to be real and do not worry about the possibility it can be negative. We also assume only the first six Hermitian modes are of interest. We are implicitly assuming that most of the energy for each dimension of the electric field is contained within these first 36 modes --6 for each dimension. If that is not true, then it is necessary to use a different set of orthonormal modes so we can use continue to use 6 modes per dimension. In eq (12) we define a weighting function $W 1$ as,

$$
W_{1}(x)=\exp \left[-\left(x / W_{1}\right)^{2}\right]
$$

Its purpose is to allow the cross-correlation process to have exact orthogonality for the fundamental propagating mode at the Fourier transform plane. We use $v(j)$ to set the phase symmetry of $T(-x)=-T(x)$.

$$
\begin{array}{rlrl}
v(j) & =\pi / 2 & j & =0,2,4 \\
& =0 & j & =1,3,5 .
\end{array}
$$

This is done because the actual filter must have a dc offset to prevent negative values. This $d c$ offset is important only to make sure that a dc signal peak in the Fourier plane does not match the signal peaks for the crosscorrelation process. We ignore the dc term in the optical filter. The positioning of the cross-correlation processes is controlled by the $u(j)$ which is set by eq (15)

$$
u(j)=\frac{2 \pi}{\lambda f} x_{f}^{u}(j) \text {. }
$$

$x^{0} f(j)$ are specified locations in the Fourier-transform plane relative to the optical axis. The strength of each cross-correlation process is controlled by the $B_{j}$ terms.

The action of the Fourier-transform lenses changes the one-dimensional field mode indicated by eq (11) to a Fourier-transformed field mode indicated in eq (16):

$$
S_{n}(u)=\frac{1}{\sqrt{\lambda f}} \int_{-\infty}^{\infty} d x e^{i u x} T(x) H_{n}(\sqrt{2} x / W) W(x) .
$$


Equation (16) has a constant phase term missing which is of no importance in our discussion. If we insert the form for $T(x)$ we get the transformed field mode

$$
S_{n}(u)=\sum_{j=0}^{5} R_{e}\left\{\exp [i v(j)] S_{n, j}(u)\right\} / C_{j},
$$

where we have the cross-correlation field mode defined in an orthonormal expansion of Hermite functions. Thus

$$
S_{n, j}(u)=\sum_{m=0}^{\infty} G_{n, j, m} m_{m}\left(\sqrt{2} u / w_{f}\right) w 2(u) / C_{m},
$$

with each mode coefficient given as

$$
G_{n, j, m}=\left(\frac{\partial}{\partial z_{3}}\right)^{m}\left(\frac{\partial}{\partial z_{2}}\right)^{j}\left(\frac{\partial}{\partial z_{1}}\right)^{n} D\left(z_{3}, z_{2}, z_{1}\right)
$$

Here we have a fundamental generator function defined as

$$
D \equiv \frac{\sqrt{2}}{w_{f} \sqrt{\lambda f}} \int d u d x e^{i u x} z\left(x, z_{1}, w\right) Z\left(x, z_{2}, w\right) Z 1\left(u, z_{3}, w_{f}\right) / w 1(x),
$$

where

$$
w 2(u)=\exp \left[-\left(u / w_{f}\right)^{2}\right]
$$

and where $\mathrm{Zl}$ is the same as $\mathrm{Z}$ with $\mathrm{u}$ replacing $\mathrm{x}$ and $\mathrm{W}_{\mathrm{f}}$ replacing $\mathrm{w}$.

We can integrate analytically over $x$ and $u$ in eq (20). First we make a set of definitions

$$
\begin{aligned}
& \frac{1}{q^{2}}=\frac{2}{w^{2}}-\frac{1}{w_{1}^{2}}, \\
& \frac{1}{p^{2}}=\frac{1}{w_{f}^{2}}+\frac{q^{2}}{4},
\end{aligned}
$$

and 


$$
w_{1} \equiv w b .
$$

The generator $D$ becomes

$$
D=\frac{\sqrt{2 \pi}}{\sqrt{\lambda f}} \frac{q p}{w_{f}} \exp \left|\begin{array}{l}
-z_{1}^{2}-z_{2}^{2}-z_{3}^{2}+2 \frac{p^{2}}{w_{f}{ }^{2}} z_{3}^{2}+2 \frac{p^{2} q^{2}}{w_{f}^{2} w^{2}}\left(z_{1}+z_{2}\right)^{2} \\
+i 2 \frac{p^{2} q^{2}}{w_{f} w} z_{3}\left(z_{1}+z_{2}\right)
\end{array}\right| \text {. }
$$

This equation form shows us what the cross-correlation process is doing. The $z_{3}=0$ case corresponds to the fundamental mode in eq (18), which corresponds to $m=0$. The desired cross-correlation process has only correlation between the $n=j$ terms. This can only happen when the relation

$$
p q=\frac{w w_{f}}{\sqrt{2}},
$$

is true. This implies

$$
\begin{gathered}
w_{f}=2 / w_{1}, \\
p=w_{f}\left(1-\left(w_{f}\right)^{2 / 8}\right)^{1 / 2},
\end{gathered}
$$

and

$$
q=w /\left[\sqrt{2}\left(1-\left(w w_{f}\right)^{2 / 8}\right)^{1 / 2}\right]
$$

Under these conditions $D$ can now be written as

$$
D=\frac{\sqrt{\pi w}}{\sqrt{\lambda f}} \exp \left[2 z_{1} z_{2}+z z_{3}\left(1-1 / b^{2}\right)+i 2 z_{3}\left(z_{1}+z_{2}\right) / b\right]
$$

When $b=\infty$, we have the ideal cross correlation, a delta function. Only $z_{3}=0$ case has no mixing between $n \neq j$ terms. Notice that the mode series are infinite. This implies a clipping error when the beam is injected into 
the optical fiber. The value $b=1.0$ corresponds to the situation that the $m=0$ mode has a width equal to the Fourier transform of the original Gaussian beam before the optical filter. The best we can do is to get a nominal minimum in the strength of the higher order modes at the Fourier plane. We assume that this is done. In the optical fiber subsection we find that the value of the width is $26.68 \mu \mathrm{m}$. This implies $b=1.19$.

There remains only one more step in this subsection, to explicitly differentiate the generator $D$ to find $G$ in eq (19). We not do this since the expressions are complex and all the details of interest have been shown and discussed except to note that the $m=1$ case is $90^{\circ}$ out of phase relative to the $m=0$ case. This situation allows a precise alignment strategy for the optical fibers. This is true because we have set up the apparatus with flat phase fronts at each of the critical locations, namely the optical filter and the Fourier-transform plane. Other locations may not allow this situation to be true. A careful analysis of other situations would be necessary to check this point.

\subsection{Effects of Beam Clipping and Optical Axis Offset between Fiber and Cross-Correlation Beam}

Here we look at the mode mixing effects due to clipping and to a shift in the optical axis of the cross-corrrelation mode compared to the optical axis of the optical fiber. Figure 6 illustrates the points. The value $d_{1}$ is the radius of the optical fiber which accepts the laser radiation, and $\varepsilon$ is the amount of relative shift of the optical axis. The critical parameter of scale is the width of the fundamental mode. We use $w=26.68$ um as that width. The value of $d_{1}$ is $150 \mu m$. This implies a distance of 5.62 times the characteristic width of the fundamental mode. This will imply a very small level of clipping when the number of modes present in the cross-correlation process is sufficiently smal1. This is the case when the value of $b$ discussed in the previous subsection is near enough to one. If we require a position adjustment accuracy of $0.5 \mathrm{\mu m}$ for the optical axis, then we would have sufficiently small error in the offset between the two optical axes. It would be about 0.1 percent or less depending on the stability of the technique for adjusting the optical axis. We look at this point in the next subsection. We now get the analytical expression for the effects of clipping and offset. 


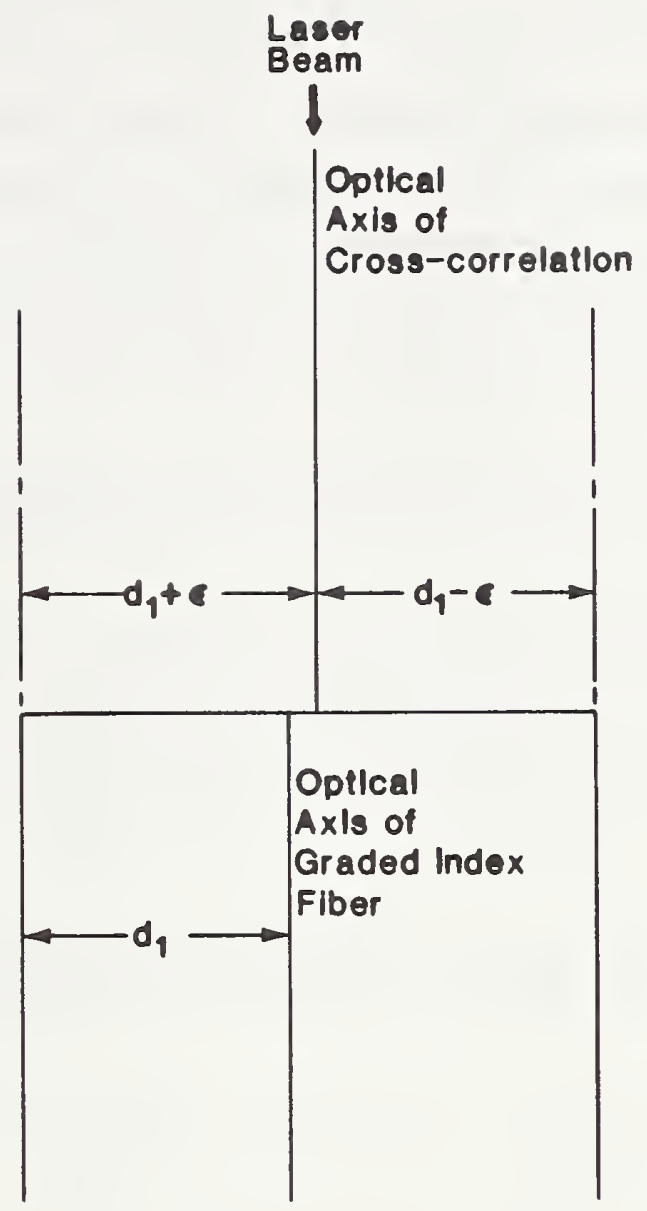

Figure 6. One-dimensional version of clipping and offset effects due to the graded-index fiber acting on a cross-correlation structure.

The one-dimensional electric field for one polarization state can be written as

$$
E(x+\varepsilon)=\sum_{m=0}^{\infty} A_{m}\left(\frac{\partial}{\partial z}\right)^{m} Z(x+\varepsilon, z, w) / C_{m},
$$

where $x$ is the coordinate perpendicular to the optical axis of the optical fiber, $\varepsilon$ is the offset of the optical axis of the cross-correlation process, $w$ is the width of the fundamental Hermite mode, $A_{m}$ is the strength of the $\mathrm{mth}$ mode, and $C_{m}$ is defined in eq (8). The $Z$ is defined in eq (8) also.

Once the radiation is launched within the optical fiber, we would have the following form for the electric field, namely 


$$
E_{c}(x)=\sum_{m=0}^{\infty} B_{m}\left(\frac{\partial}{\partial z_{1}}\right)^{m} Z\left(x, z_{1}, w\right) / C_{m} .
$$

The only change between eqs (27) and (28) is the strength coefficients. We are particularly interested in the $B_{0}$ term. It provides all information about the cross-correlation process and about the particular orthonormal mode. The ideal situation is no offset error and no error due to clipping. $B_{0}$ is given as

$$
B_{0}=\sum_{m_{1}=0}^{\infty} A_{m_{1}}\left(\frac{\partial}{\partial z}\right)^{m_{1}} D_{2}\left(z, 0, d_{1}, \varepsilon\right) / C_{m_{1}}
$$

where $D_{2}$ is defined as

$$
D_{2}\left(z_{1} z_{1}, d_{1}, \varepsilon\right)=\frac{\sqrt{2}}{w} \int_{-d_{1}}^{d_{1}} d x z(x+\varepsilon, z, w) Z\left(x, z_{1}, w\right)
$$

If we make the definitions

$$
a \equiv \sqrt{2} \varepsilon / w, b=\sqrt{2} d_{1} / w, u=\sqrt{2} x / w,
$$

and

$$
c=z-a / 2,
$$

then $D_{2}$ is given as

$$
D_{2}\left(z, 0, d_{1}, \varepsilon\right)=\exp \left[-a^{2} / 4-a z\right] D_{3}(b, c)
$$

where $D_{3}$ is defined as

$$
D_{3}(b, c)=\int_{-b-c}^{b-c} \exp \left[-u^{2}\right] d u .
$$

If we make the approximation that $b$ is very large and $a$ is small, then the relationship between $B$ and $A$ is

$$
B_{0} \cong A_{0}-a A_{1} / 2
$$


The key fact to notice here is that an error in the optical axis can be significant because the strength of $A_{1}$ is comparable to $A_{0}$. First we note the fact that $A_{1}$ is $90^{\circ}$ out of phase with $A_{0}$. This means the magnitude of $B_{0}$ is proportional to $a^{2}$. If $a=0.03$ we would have a 0.1 percent error due to alignment. This implies a positioning accuracy of $0.5 \mu \mathrm{m}$. Subsection 3.7 describes how this can be achieved. If we make the approximation that a is zero and $b$ is large, then the relationship between $B$ and $A$ is

$$
B_{0} \cong A_{0}\left(1-e^{-b 2} / b\right)-e^{-b 2} \sum_{m=0}^{\infty} A_{m+1}\left[H_{m}(b)-H_{m}(-b)\right] / C_{m+1} \text {. }
$$

Here we simply notice that $b$ needs to be chosen so that the highest $A_{m}$ modes are less than 1 percent of the $A_{0}$ mode. The choice of a graded-index optical fiber of $150 \mu \mathrm{m}$ radius and $w=26.68 \mu \mathrm{m}$ allows $b=5.62$. As 1 ong as $m$ is 1 ess than 30 modes we have no significant clipping effect.

3.7 Design of a High Precision Positioning Device to Center the Optical Axis of Each Fiber to the Optical Axis of the Cross-Correlation Process

Figure $7(a)$ shows the nominal mounting of the optical fibers shown as $C$ so that the piezoelectric material can be used to get precision adjustment of each fiber's optic axis. The piezolelectric material is indicated by $A$ and B. They each have the dimensions of $0.1 \mathrm{~mm}$ thick, $1.0 \mathrm{~mm}$ wide, and $20 \mathrm{~mm}$ long. There are six such units for each 3-by-3 array of optical fibers. To support these units, there is a ceramic frame with notches to allow the piezoelectric material to lengthen at least $50 \mathrm{um}$. The outside pair of notches are placed with center of $\pm 5 \mathrm{~mm}$ relative to the center notch. The width of the notches is $0.12 \mathrm{~mm}$. The depth of the notches is $0.2 \mathrm{~mm}$. The notches run from the front edge to a depth of $2.2 \mathrm{~mm}$. The frame has the dimensions, $d_{8}=1.0$ $\mathrm{mm}, d_{1}=30 \mathrm{~mm}$ and $d_{4}=d_{5}=21.9 \mathrm{~mm}$. This frame is constructed so that front and back edges are strictly parallel. This frame is rigidly attached to the electroform in the back. The piezoelectric material is placed in the frame. The remaining big step is to get precision in the placement of the optical axes of the nine fibers. We need the coordinates to be accurate to \pm 1.0 um to the design positions. If we can get that accuracy, then it will be possible to use the piezoelectric material to place the optical axes to \pm 0.2 um accuracy, which would eliminate the offset error. 


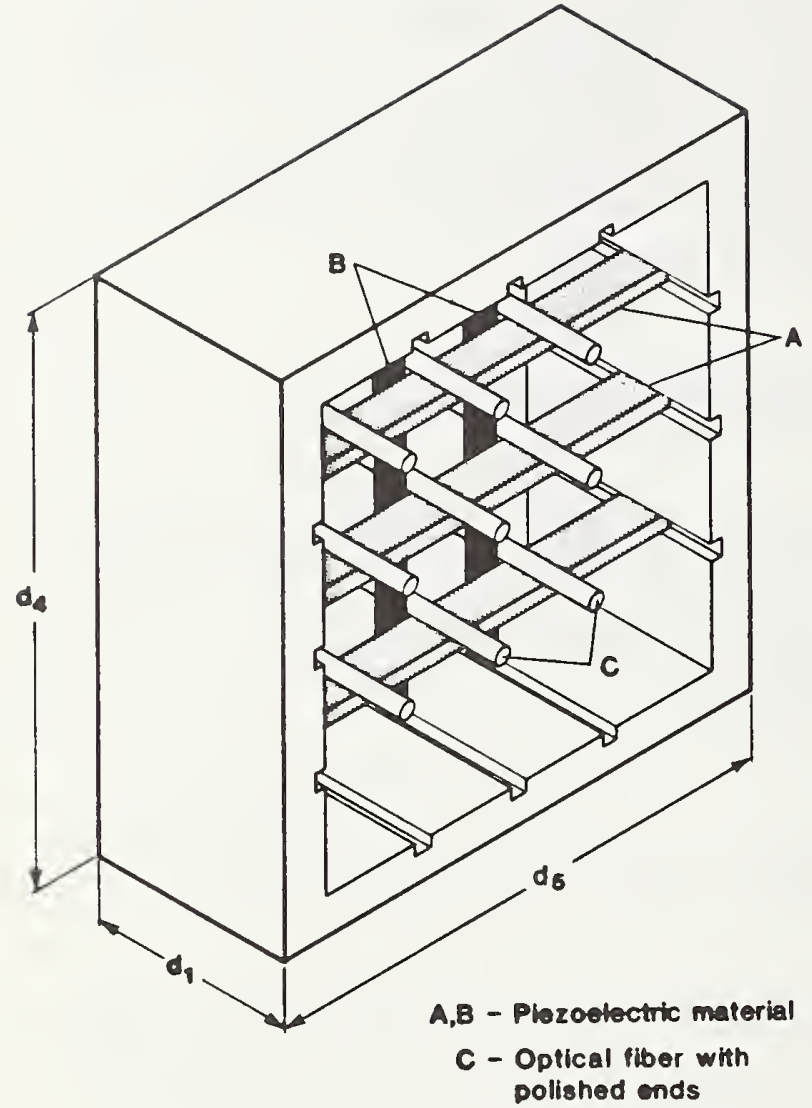

(a)

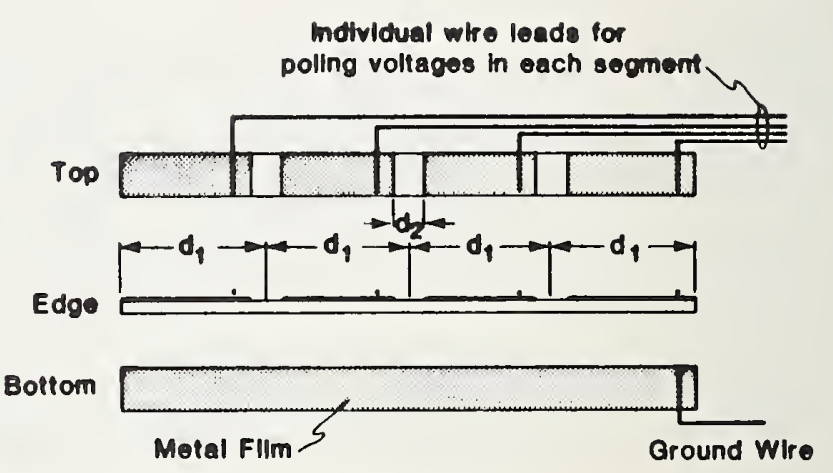

(b)

Figure 7. (a) Mounting of piezoelectric material to allow two-dimensional position adjustment of the optical fibers. (b) Detail of a piezoelectric material.

We presume a jig to allow placement of the nine optical fibers to $x$ and $y$ axis coordinates of $\pm 5 \mathrm{um}$ for each fiber relative to the center fiber of the 3-by-3 array of fibers. The accuracy of this placement must be at least $\pm 0.5 \mathrm{~mm}$. We suggest that the jig can be constructed using a set of holes constructed by electroformed material with the nine hole sizes made 1 um greater (301 $\mu \mathrm{m})$ than the outside diameter for the uncoated part of the fiber. We believe the fiber can be constructed with a consistent diameter within 1 um. Assuming this is true, the $\mathrm{jig}$ is used to align the 3-by-3 array of fibers. The back electroformed unit becomes a second jig with one modification, namely that the hole size is $350 \mathrm{~mm}$. It becomes a part of the apparatus. The precision jig is temporary. It would be located on the front side of the 3-by-3 
array at a distance of $40 \mathrm{~mm}$ from the back jig. It is also attached by using a solvent to dissolve the sheath of the nine fibers and then inserting the fibers into the precision jig. The conical shape of the hole in this jig would expand toward its back. A number of precision spacers of 1 um difference in length is used to align the front jig relative to the back jig and to render the two jigs rigid to each other. The thickness of both jigs would be about $0.5 \mathrm{~mm}$ so they are sufficiently rigid. Notice there is a $10 \mathrm{~mm}$ separation between the frame and the front jig. This should be enough distance to allow the gluing of the piezoelectric materials to the optical fibers and to the frame. The glue or epoxy must provide a rigid mechanical bond for the optical fibers and for one side of each piezoelectric slab. To make the unit insensitive to mechanical vibration and just allow the slab to lengthen or shrink freely, the other side of the slab needs to be attached just on the edge of the notch with a rubber type of material--perhaps silicone rubber.

There remains one final construction detail, namely how to cut the fibers to a proper length and polish them. We use a new jig made like the cover plate indicated in figure 5 where the holes are $350 \mu \mathrm{m}$ in diameter and where the conical shape of the holes expands toward the front. We remove the jig with the $301 \mathrm{\mu m}$ holes and rigidly attach the new jig to the frame. We now have optical fibers sticking out about $10 \mathrm{~mm}$ above the new jig. The front part of the jig is covered with paraffin wax. This will make the unit sufficiently rigid to allow the cutting and polishing of the excess optical fibers. The 3-by-3 array unit is mounted in a lathe with the rotation axis parallel to the optical fiber axes. Using a thin one-sided wedge angle tool, we cut as close as posible to the front surface of the jig. We then polish the unit so that the nine optical fibers are optically flat relative to each other to at least $1 \mu \mathrm{m}$. Once this step is done, then the remaining wax is removed by a solvent and by ultrasonic cleaning. The new jig is now removed. Figure $7(b)$ shows the detail for the metal films and wire leads on the piezoelectric material. The bottom side is covered with a metal film to act as the ground plane. A ground wire is attached with a conducting epoxy. The top side is covered with metal film except for three regions of width $\mathrm{d}_{2}=1 \mathrm{~mm}$ where there is no film. The intervals between these regions are $d_{1}=5 \mathrm{~mm}$. There are four individual wires attached with conducting epoxy to each film segment. The five wires pass through the frame of figure $7(a)$ through notches (not 
$A, C$ - Giase suport for theer

B - Glass support for tapered position of flber

$D$ - Electroform of nickel with holes to all fibers to be hold

E - Electroform with holes to shield out extraneous radiation

F - Polished ends of optical flber

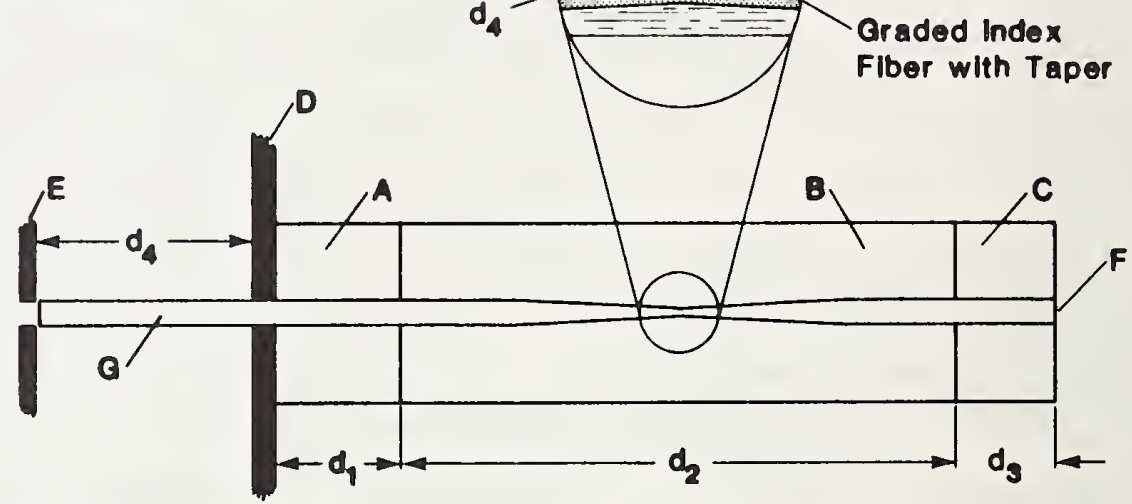

Figure 8. Side view of one optical fiber showing the individual supports.

shown in the figure) in the front surface of this ceramic frame. These wires provide the means for getting a dc electric field onto each segment of the piezoelectric material.

There remain two primary points of discussion, namely the reason for the choice of $30 \mathrm{~mm}$ for the length of the fiber and the dynamic range of the movement of the fiber due to the piezoelectric effect. We discuss the effects of four potential voltages on each piezoelectric strip. To first approximation, we can expect a sum of linear shifts. A more exact analysis would produce a two-dimensional shift pattern. To understand this requires assembly of the unit and testing of the unit. We now address the primary point.

Figure 8 shows how a single fiber is mounted to the support base. Figure $7(a)$ shows how the array of fibers are mounted. The key point to note is that this is a cantilevered beam configuration. This means that we can look up the formulas for the displacement of the end of the beam under two conditions--gravity and external force at the end. The gravity force is a distributed force. Its effect, which is proportional to the fourth power of the length, depends on the flexibility of the fiber. A $30 \mathrm{~mm}$ length implies a sag of $5 \mu \mathrm{m}$ at the end. Under these conditions a force of 1 gram will imply a displacement proportional to the third power of the length, namely $310 \mu \mathrm{m}$. In 
contrast, a $20 \mathrm{~mm}$ length implies as sag of $1 \mathrm{\mu m}$ and a corresponding displacement of $92 \mathrm{\mu m}$. The former results assume no significant resistance to movement of the end. The pair of piezoelectrics act to support the end of the fiber and to control its position with no important resistance to the two transverse motions.

To address the final primary point, we need to specify the piezoelectric material. With our thickness of $0.1 \mathrm{~mm}$ and with the application of $100 \mathrm{~V}$, we can develop an electric field of $1 \mathrm{MV} / \mathrm{m}$. If we have a length of piezoelectric material of $5 \mathrm{~mm}$, a mechanical electric coupling efficiency of 0.6 , and piezoelectric constant of $270 \times 10^{-12} \mathrm{~m} / \mathrm{V}$, we can get a displacement of $0.8 \mathrm{um}$. This implies a range of adjustment of $\pm 0.4 \mathrm{um}$ which is adequate for fine tuning the optical axis of each fiber. It will be necessary to temperature control this 3-by-3 array so that the temperature effects in the piezoelectric material become unimportant. The best way would be to temperature control the entire unit beyond the Fourier plane to some fixed value such as $30^{\circ} \mathrm{C}$ with an accuracy of $0.1^{\circ} \mathrm{C}$. In this way we would guarentee the dimensional stability of all the parts including the piezoelectric material. In addition we would control the temperature dependence of the responses of all the cietectors.

\subsection{The Mode Attenuator--The Tapered Optical Fiber}

Figure 8 shows the structure of the tapered optical fiber. We address first the structural details, next the construction details, and finally the mode stripping process.

We look at the structure from the left to right. $E$ is the cover plate at the Fourier-transform plane. $G$ is the graded-index fiber. It goes from the cover plate to the back support indicated by $C$. $D$ is the support, which acts as the rigid locator for the optical fiber. The glass supports $A$ and $C$ are used to hold the fiber and are used to prevent the index matching fluid within support B from leaking out. The optical fiber is tapered from the $150 \mathrm{~mm}$ radius to about $5 \mu \mathrm{m}$ and back to $150 \mu \mathrm{m}$ within the length of the support $B$. The $5 \mu \mathrm{m}$ location is near the center of support $B$. Both ends of the fiber are polished. The nominal dimensions are $d_{4}=30 \mathrm{~mm}, d_{1}=10 \mathrm{~mm}, d_{2}=50 \mathrm{~mm}$, and $d_{3}=10 \mathrm{~mm}$. The diameter of the supports $A, B$, and $C$ must be less than $9 \mathrm{~mm}$ with a capillary of about 400 um diameter. 
The sugyested construction details are to first mount a $300 \mathrm{~mm}$ long fiber inside support A with $40 \mathrm{~mm}$ in front of the support. Use an index matching glue to do the mounting. Insert the fiber into structure $B$ and suspend the unit with support $A$ holding up the fiber unit. To prevent support B from falling off, clamp the bottom end of the fiber with a metal clamp. The unit should now have a space of about $180 \mathrm{~mm}$ between mount $A$ and the support $B$ where we can see the optical fiber. Using a $\mathrm{CO}_{2}$ laser, we heat a segment of fiber. The weight of the support $B$ and the metal clamp will stretch the optical fiber to form a taper. The length and pith of the taper is controlled by how far the stretch can take place. By having a jig which will stop the fall of support $B$ and which will make sure there is no twisting or turning of the support $B$, we can control the final size of the taper. This process is reported until the fiber is the correct size. After the glass fiber is so contructed, we put a drop of index matching fluid at the top of the support where the optical fiber enters, then we slide the support B up to support $A$ and glue them together. This action is necessary to protect the taper and to support the optical fiber. It is desirable to make the start of the melt as close as possible to the $A$ unit. During this assembly it is necessary to make sure that the index matching fluid fills the support $B$. After this step is completed, the support $C$ is added and glued to the support $B$ and to the optical fiber. At this stage the optical fiber is now in a rigid mount and has been protected. The next step in construction is to polish the optical fiber at the exit end.

Within this construction process, there are several points to notice: (1) there are nine of these units for each 3-by-3 array and there are four such arrays, (2) the unit $A$ is glued to the support structure $D$ of the array. At this point, the optical fiber is placed $40 \mathrm{~mm}$ in front of the unit $D$. Figure 8 shows the optical fiber terminating before the cover plate. The construction of the piezoelectric unit left the optical fiber flush with the front of the cover plate. To make the fiber terminate before the cover plate, we add a spacer of the thickness of the cover plate. The cover plate is coated with a radiation absorbing material so that there is minimum stray radiation getting to the optical fiber. Also it is desirable to orient the cover plate $E$ so that the conical shape of each hole in the electroform expands toward the optical fiber. 
We now have some analytical points to make. The mode-count number $V$ in one-dimension for optical fibers is

$$
V=n b \pi(2 \Delta)^{1 / 2} \lambda
$$

where $\Delta=(\Delta n) / n, b$ is the radius of the optical fiber, $n$ is the index of refraction of the glass and $\lambda$ is the wavelength of laser radiation. If we assume $\Delta=0.001, b=4.75 \mu \mathrm{m}, n=1.5$, and $\lambda=1.0 \mu \mathrm{m}$, we find that $V=1$, which means that we have only one propagating mode present in the optical fiber for each polarization state. In contrast, if we assume $b=150 \mu \mathrm{m}$, we find that $V=32$. This means that there are about 2000 modes in the fiber.

Assuming at the start a uniform rod of length $1_{0}$, and at the end a uniform taper, both in shrinking and expansion phase, the new length, 1, of the tapered optical fiber compared to the old length, $1_{0}$, is given as

$$
1_{0}=1\left(1-a+a^{2} / 3\right),(37)
$$

where $a$ is the ratio of the difference between the initial radius of the fiber to the final radius divided by the initial radius. If we use for the intial length $1_{0}=10 \mathrm{~mm}$ and $a=145 / 150$, we get $1=29.0 \mathrm{~mm}$ or a change of length of $19 \mathrm{~mm}$. The taper angle is $2 \mathrm{r} / 1 \cong 2 \mathrm{deg}$. Equation (37) is derived from the assumption that glass material is conserved.

It is convenient at this time to add one more formula to this list, namely the one that specifies the width $w_{0}$ of the fundamental mode of the graded-index fiber.

$$
w_{0}=(\lambda a / n \pi)^{1 / 2}(1 / 2 \Delta)^{1 / 4}
$$

Using the values given for eq (36) we get $w_{0}=26.68 \mu \mathrm{m}$ for $\mathrm{a}=150 \mu \mathrm{m}$ and $w_{0}=4.75 \mu \mathrm{m}$ for $a=4.75 \mu \mathrm{m}$. A change in the wavelength of 10 percent causes a 5 percent change in the width of the fundamental mode. To get highest accuracy in the electric field measuring apparatus, it is necessary to match the optical filter cross-correlation width $w_{0}$ at the particular wavelength. A calculation just like the beam clipping would show the mode mixing error due 
to beamwidth mismatch of 1 percent would be 2 percent error. This is not to be ignored. This fact basically implies that each optical filter will be unique for each wavelength.

The ideal procedure is to have the support glass made of a radiation absorbing material so we can be sure all modes that do not satisfy the support condition given by the radius of the graded-index fiber will be absorbed as quickly as possible and the propagating modes will not be absorbed. The delicate point is to get the neck of the taper long enough to allow the modes above the fundamental mode to be attenuated and yet not cause the structure to break during the fabrication process. The above construction procedure is expected to do the job. What is very critical is to make sure that the stretching process keeps the graded-index structure cylindrically symmetric over the entire length of the taper. In this way we can be sure the two fundamental modes are not significantly attenuated. It should be obvious to the reader that this taper method for attenuation of modes depends intimately on the form of the modes. The primary loss is the energy exterior to the core. By having a Gaussian fundamental mode, we tend to place the energy of the undesired modes exterior to the fundamental modes and hence can get mode attenuation with minimal mode mixing. One final point of this subsection. It is not necessary to get the neck to 5 um radius. What is necessary is to get the neck small enough that the fundamental mode still propagates and the other modes are attenuated because they are not being sustained by the core. This condition happens as long as the radius a is less than $9.49 \mu \mathrm{m}$. We suggest that the best range has a equal to about $6 \mu \mathrm{m}$. In this way we do not have the fiber too small and yet make the neck long enough to attenuate undesired modes.

\subsection{The Polarization Analysis Process--A Calcite Crystal}

If the optical fiber and the tapering process do not cause more than 10 percent mixing between the two polarization states, then it is possible to structure the apparatus so that polarization can be accurately measured for each of the original laser modes. A calibration of the apparatus is necessary to determine the level of the polarization mixing process. For illustration we assume that the original $300 \mu \mathrm{m}$ yraded-index fiber has a polarization mixing rate of 0.1 percent per centimeter for the fundamental mode in the fiber. 
We assume the taper process increases this mixing to 1 percent per centimeter. Under these conditions, the actual mixing would be 4 percent which would be tolerable. The actual way to attain this situation will require that the graded-index fiber be made as strain-free as possible and as cylindrically symmetric as possible. One way to estimate how well this can be done is to note that current single mode fibers with such cylindrical symmetry can be made with a birefrigence of $5^{\circ}$ per meter. This implies a mixing of 0.02 percent per centimeter which is well within our calculation.

If we have a calcite crystal which has been cleaved and polished with the principal section normal to the cleaved surfaces, we can get a separation of the ordinary and extraordinary polarizations. The separation angle is $6.2^{\circ}$. A $5 \mathrm{~mm}$ thick slab will separate a laser beam into two beams $0.54 \mathrm{~mm}$ apart. The original $27 \mu \mathrm{m}$ width beam will expand to $65 \mu \mathrm{m}$ width over this distance. Thus the beam separation is 8.3 beamwidths which is adequate for separation. There is one concern in the use of calcite: it is brittle. The assembly process will need to account for this fact. It may be necessary to make the $C$ structure nine separate calcite crystals mounted in a plastic frame which is then mounted as shown in figure 9.

Figure 9 shows the structure of the assembly of the calcite crystal as well as the other parts of the $3-b y-3$ array. There are six points to notice:

1. The calcite crystal is either 20 by $20 \mathrm{~mm}$ or is nine crystals of 4 by $4 \mathrm{~mm}$ in size.

2. The glass rods and calcite are encased by two nickel electroforms and a rectangular aluminum frame of length $d_{2}=75 \mathrm{~mm}$ and cross section of $\mathrm{d}_{1}=25 \mathrm{~mm}$.

3. The ends of each glass rod are coated with a light absorbing paint except at the optical fiber.

4. The calcite is matched to the optical fiber by index matching fluid such as mineral oil.

5. The back piece of electroform, $F$, has nine circular holes of $2 \mathrm{~mm}$ diameter. These are needed to allow the detectors to see the laser radiation from each beam.

6. To eliminate mixing of radiation, the space between the glass rods is filled with a light-absorbing material. If the unit works as intended, 


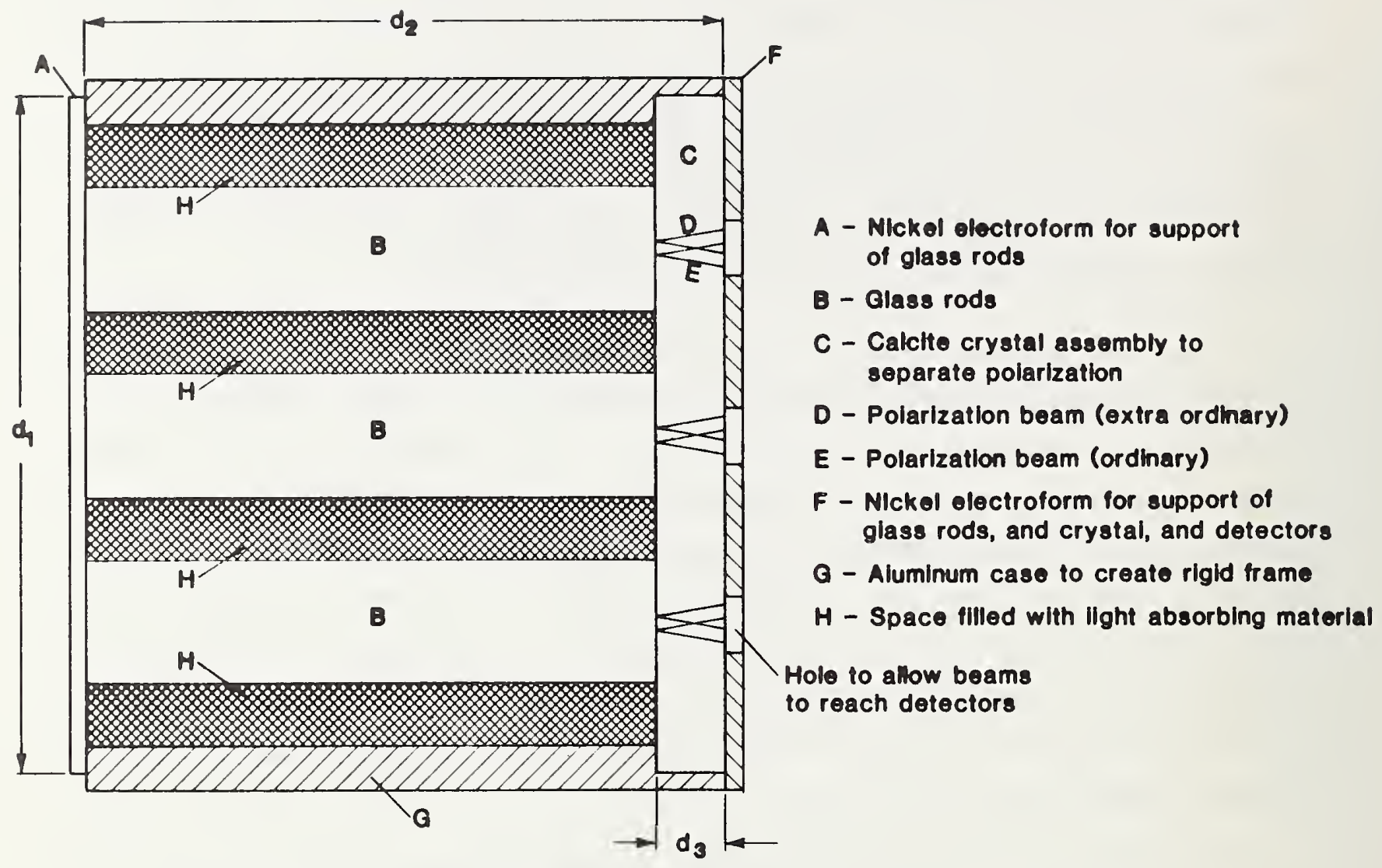

Figure 9. Polarization separation and support structure.

then it is desirable to use epoxy for mechanical rigidity. For testing purposes, it may prove better to use a liquid such as water or oil with a dye in it. The liquid has the advantage that it can be removed if the unit needs to be repaired. A high specific heat material such as water will attenuate significantly the temperature fluctuations of the environment acting on the 3-by-3 array.

If the polarization mixing is greater than 10 percent, then it will be necessary to do the polarization analysis just after the optical filter with a very good quality polarizer. The effects of the phase shift will need to be considered. In this case, the polarization measurement would be done by repeating the measurement with the orientation of a linear polarizer shifted by $90^{\circ}$ between each measurement. 
We now need to measure the strength of each Gaussian beam and its phase function as a function of time.

If it is desirable to measure the phase, then it will be necessary to use these 72 beams and mix them with a reference beam which has phase dependence that is known in some sense to produce a signal that allows determination of the relative phase of these 72 beams to the reference beam. The reference beam could come from a sampled beam obtained at the optical axis of the apparatus with a single mode fiber. This sample is gotten at the Fourier plane with due consideration of various propagation distances.

For now, we assume the phase measurement process is not necessary. Under this assumption, figure 10 shows a possible configuration for the detectors used to measure the strength of a Gaussian beam. We see the calcite assembly designated as $C$, the holder for the detectors designated as $B$, and the holder for the assembly and the glass rods designated as $A$. The detectors are located in the holes made in $A$. The detectors have a width of $0.5 \mathrm{~mm}$ and are

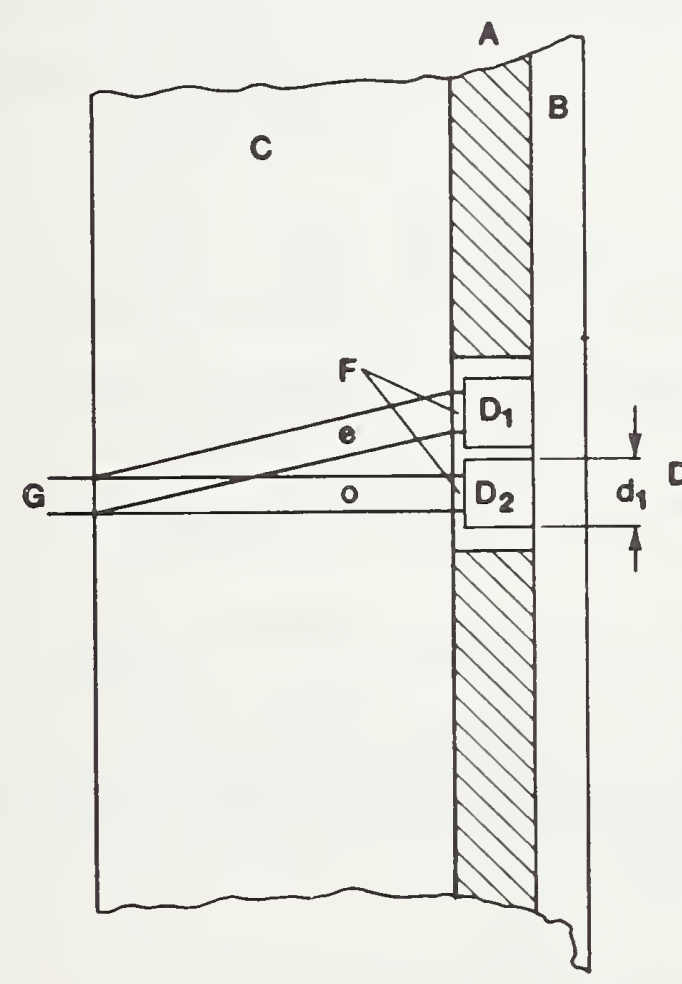

\section{A - Electroform support with holes}

B - Electronic circuit board to hold detectors

C - Calcite crystal assembly

F - Index matching fluid between calcite and detector

$D_{1}, D_{2}-$ Two detector assemblies to measure laser power

G - Optical fiber

- Extraordinary beam

o- Ordinary beam

Figure 10. The case where only laser power is measured. 
centered for each beam. These detectors are matched to the calcite with index matching fluid so that there is minimal reflection. The key reflection is the one from the surface of each detector. It propagates back to the front surface of the calcite assembly where it is absorbed by the paint on the back surface of the glass rods. This process should minimize the effects of the reflected beams. The detectors would be either silicon for the visible light range or pyroelectric detectors if the power levels are high enough.

Once the power is measured, the current or voltage is then captured by some form of sample and hold circuitry for the A/D processing. Selection of that circuitry is dependent on the time resolution desired. Because the detectors are small, we can expect resolution of signals at faster than nanosecond rates.

4. Realization of Nonlinear Filter Using Abstract Mathematics

4.1 Overview

In this section we develop the mathematical expressions necessary for the computer programs. These programs are used to generate the optical filter. Subsection 4.2 generates the exact equation and subsequent approximations using all the various constraints due to the fabrication process. Subsection 4.3 examines the part of $T(x)$ which has the spatial dc characteristic of the fabrication process. Subsection 4.4 examines what happens due to the $0.15 \mu \mathrm{m}$ error in positioning the cel1. Subsection 4.5 examines the antisymmetric part that is nonlinear. Subsection 4.6 examines the antisymmetric part that is quasilinear. Finally, subsection 4.7 discusses two strategies for fabrication of the two-dimensional optical filter to establish the most accurate one.

\subsection{Exact Equation Incorporating the Constraints of Fabrication}

We assume that the separability into one-dimensional structures is sufficient. Under these circumstances the resulting pattern of a single mode at the Fourier-transform plane is given as

$$
S(n, u)=\sum_{r=1}^{4} S(n, u, r)
$$


Here

$$
S(n, u, r)=\frac{1}{\sqrt{\lambda f}} \int_{-\frac{L}{2}}^{\frac{L}{2}} d x H_{n}(x) T[x, a(r), b(r)] \exp (i u x) .
$$

We have formed four types of radiation patterns due to the structure of the holes in the optical filter. $T(x, a(r), b(r))$ gives the pattern for each type. Equation (42) and others below will define this function. For now notice the $H_{n}(x)$, this represents the $n$ mode of the orginal orthonormal decomposition of the electric field at the optical filter. This $n$ mode is like the Hermite mode but does not have to be exactly the same. We assume that $n$ ranges from 1 to 6. Also notice that the range in integration is $-(L / 2)$ to $+(L / 2)$. This is due to the finite aperture of the optical filter which has $L=70 \mathrm{~mm}$.

Because we are only interested in the fundamental fiber mode, we multiply the expression in eq (40) by that in eq (41), namely

$$
\frac{\sqrt{2}}{\sqrt{ } \pi w_{m}} \exp \left\{-\left[(u-u(r)) / w_{m}\right]^{2}\right\},
$$

and integrate over $u$ to get the strength of each radiation pattern in the fundamental mode. There is an approximation in this expression which is less than 0.2 percent. We get

$$
S 1(n, k, r)=\sqrt{\frac{2}{\lambda f}} \sum_{\ell=-N+1}^{N} H_{n}\left(y_{\ell}\right) B\left(z_{\ell}\right) \exp \left[i u(k) y_{\ell}-\left(w_{m} y_{\ell} / 2\right)^{2}\right]
$$

We have a large number of definitions to note:

First we give the form of $T$, namely

$$
T(x, a(r), b(r)] \equiv \sum_{\ell=-N+1}^{N}\left[\theta\left(x-x_{\ell}-a_{\ell}(r)\right)-\theta\left(x-x_{\ell}-b_{\ell}(r)\right)\right],
$$

where we have below the step function, the centroid coordinate, the half width of each hole in the optical filter, and the nominal stepping interval between the holes.

$$
\theta(x)=1 \quad x \geqslant 0 \quad \theta(x)=0 \quad x<0,
$$




$$
\begin{aligned}
& y_{\ell} \equiv x_{\ell}+\left(a_{\ell}(r)+b_{\ell}(r)\right) / 2, \\
& z_{\ell} \equiv\left(b_{\ell}(r)-a_{\ell}(r)\right) / 2 \text {, and } \\
& x_{\ell} \equiv D 4(2 \ell-1) .
\end{aligned}
$$

The width of the fiber in $u$ coordinates is related to the actual fiber mode width in position coordinates of the Fourier plane as

$$
w_{m}=2 \pi W_{\text {fiber }} / \lambda f=0.168(\mathrm{~mm})^{-1} \text {. }
$$

Some values that are specified by the conditions of design are:

$$
\begin{aligned}
& u(k)=N(k) \Delta u \\
& \lambda f=1.0(\mathrm{~mm})^{2} \\
& \Delta u=2 \pi \Delta \hat{x} / \lambda f=0.0898(\mathrm{~mm})^{-1} \\
& \Delta u_{c e 11}=2 \pi \Delta \hat{x}_{\text {cel } 11} / \lambda f=31.42(\mathrm{~mm})^{-1} \\
& \Delta x=\lambda f / L=\lambda f /(4 N D 4)=(1 / 70) \mathrm{mm} \\
& \Delta \hat{x}_{\text {ce } 11}=\lambda f / \Delta x_{\text {cel1 }}=5 \mathrm{~mm} \\
& \Delta x_{\text {ce } 11}=200 \mu \mathrm{m} .
\end{aligned}
$$

The cell set is defined by the $200 \mu \mathrm{m}$ cell size. This implies a spacing in the Fourier plane of $5 \mathrm{~mm}$. The second spacing is defined by the finite aperture.

Some further definitions or consequences of constraints in the design are:

$$
\mathrm{D} 4=\mathrm{D} 0 / 2=5 \mu \mathrm{m},
$$




$$
\begin{aligned}
& D 0=10 \mu m, \\
& N=3500,
\end{aligned}
$$

and

$$
W_{\text {fiber }}=26.68 \text { for } \lambda=1.0 \mu \mathrm{m} \text {. }
$$

DO is the fundamental maximum spot size of the optical filter and $N$ is the total number of spots available in a $70 \mathrm{~mm}$ width filter. Wfiber will change according to eq (38) as the wavelength is changed. For maximum accuracy, each new wavelength will require a new spatial filter. $B\left(z_{\ell}\right)$ is given and approximated to

$$
\begin{aligned}
& B\left(z_{\ell}\right)=2 \sin \left[u(k) z_{\ell}\right] / u(k) \\
& \doteq 2 z_{\ell}\left[1-\left(u(k) z_{\ell}\right)^{2 / 6]} .\right.
\end{aligned}
$$

The term that is cubic in $z_{\ell}$ can be a 6 percent correction to the filter so it must be retained.

The individual strengths are summed to produce the net strength of the fundamental mode, namely

$$
S 1(n, k)=\sum_{r=1}^{4} S 1(n, k, r)
$$

At this point, we specify the $a_{\ell}(r), b_{\ell}(r), z_{\ell}$, and $y_{\ell}$. We use table 1 to specify these quantities. The TYPE label notes the following: $D C$ is the necessary offset to prevent signal at the hole from becoming negative, $A C$ is the desired signal which can be negative, El is due to the error in cell placement affecting the offset, and $E 2$ is due to the error in cell placement affecting the desired signal. 
Table 1.

\begin{tabular}{llllll}
\hline TYPE & $\Gamma$ & $a_{\ell}(r)$ & $\mathrm{b}_{\ell}(r)$ & $z_{\ell}$ & $y_{\ell}{ }^{-x} \ell$ \\
$D C$ & 1 & $-D 4$ & 0 & $D 4 / 2$ & $-D 4 / 2$ \\
$A C$ & 2 & 0 & $g_{\ell}$ & $g_{\ell} / 2$ & $g_{\ell} / 2$ \\
E1 & 3 & $\varepsilon_{\ell}-D 4$ & $-D 4$ & $-\varepsilon_{\ell} / 2$ & $\varepsilon_{\ell} / 2-D 4$ \\
$E 2$ & 4 & $g_{\ell}$ & $g_{\ell} \varepsilon_{\ell}$ & $\varepsilon_{\ell} / 2$ & $g_{\ell} \varepsilon_{\ell} / 2$ \\
\hline
\end{tabular}

There are some symmetries to note relative to $\ell$ and $-\ell+1$, namely

$$
\begin{gathered}
x_{-\ell+1}=-x_{\ell}, \\
g_{-\ell+1}=-g_{\ell}, \\
\varepsilon_{\ell} \leqslant 0.15 \mu m=0.03 \text { D4 }, \\
\left|g_{\ell}\right| \leqslant D 4 .
\end{gathered}
$$

The above also shows the restrictions in size of the error $\varepsilon$ and the range of the desired signal.

The beam turning is specified by the $N(k)$. It has the following values and properties. Here $k$ ranges from -6 to +6 .

$$
\begin{aligned}
& N(-k)=-N(k) \\
& N(0)=0 \\
& N(k)=440,615,790,965,1140, \text { and } 1315
\end{aligned}
$$

for $k=1$ to 6 . These values are chosen to allow the necessary physical spacing of the optical fibers and their associated equipment as well as avoiding resonances due to the cell placement errors. They occur at multiples of 350. The first mode correlation, $N(1)$, is placed far enough so that the signal on the optical axis has no significant contribution to that correlation. $N(2)$ is spaced far enough from $N(1)$ so that there is less than 1 percent mixing between the $N(2)$ and $N(1)$ terms.

The remaining symmetry relationships are shown in table 2. 
Table 2.

$\begin{array}{lll}r & z_{-} \ell+1 & y_{-\ell+1} \\ 1 & D 4 / 2 & -y_{\ell}-D 4 \\ 2 & -g_{\ell} / 2 & -y \ell \\ 3 & -\varepsilon_{-\ell+1 / 2} & -y_{\ell}-2 D 4+\left(\varepsilon_{\ell}+\varepsilon_{-\ell+1}\right) / 2 \\ 4 & \varepsilon_{-\ell+1} / 2 & -y_{\ell}+\left(\varepsilon_{\ell}+\varepsilon_{-\ell+1}\right) / 2\end{array}$

This completes the yeneral specification of the mathematics for the expected field strengths due to the four possible radiation patterns. We are now ready to look at those individual patterns to assess what they will do to the signal at each fiber.

\subsection{Evaluation of the dc Part}

The dc part is symmetric to better than 0.1 percent. Thus we can simplify the dc strength to the form in eq (52) where the $y_{\ell}$ and $z_{\ell}$ are defined in table 1 with $r=1$.

$$
\begin{gathered}
S I(n, k, 1) \doteq \frac{\sqrt{2}}{\sqrt{\lambda f}} \sum_{\ell=1}^{N} H_{n}\left(y_{\ell}\right) B\left(z_{\ell}\right)\left[e^{i u(k) y} \ell+(-1)^{n+1} e^{-i u(k) y} \ell\right] \\
\star \exp \left[-\left(w_{m} y \ell^{\left./ 2)^{2}\right]} .\right.\right.
\end{gathered}
$$

The key point to note about this expression is that the signal strength at each $k$ location is the Fourier transform of the $n$th mode. For $k$ not equal zero, this strength is less than 0.1 percent of the peak for the signal of interest and hence is ignored.

\subsection{Evaluation of the Cell Positioning Error}

The two cell positioning error terms are shown in eq (53) and (54) below with $r=3$ and $r=4$, respectively.

$$
\begin{gathered}
S 1(n, k, 3)=\frac{\sqrt{2}}{\sqrt{\lambda f}} \sum_{\ell=1}^{N} H_{n}\left(y_{\ell}\right)\left[-\varepsilon_{\ell} e^{i u(k) y} \ell-(-1)^{n+1} e^{-i u(k) y_{\ell}} \varepsilon_{-\ell+1}\right] \\
\star \exp \left[-\left(w_{m} y \ell^{\left./ 2)^{2}\right]}\right.\right.
\end{gathered}
$$




$$
\begin{gathered}
S I(n, k, 3)=\frac{\sqrt{2}}{\sqrt{\lambda f}} \sum_{\ell=1}^{N} H_{n}\left(y_{\ell}\right)\left[\varepsilon_{\ell} e^{i u(k) y_{\ell}}+(-1)^{n+1} e^{-i u(k) y_{\ell}} \varepsilon_{-\ell+1}\right] \\
\star \exp \left[-\left(w_{m} y_{\ell} / 2\right)^{2}\right]
\end{gathered}
$$

We notice here the error is 3 percent of the basic term and is further reduced since both terms are basically the Fourier transform of the fundamental mode. This is very weak at the locations where $k \neq 0$. The resonance due to cell positioning error is handled by having $N(k)$ not located at those cell induced resonances. Thus we neglect these error terms.

\subsection{Evaluation of the Antisymmetric Nonlinear Part}

The strength that has the desired signal is the $r=2$ case of table 1 and it has the form given by eq (55).

$$
\begin{gathered}
S 1(n, k, 2)=\frac{\sqrt{2}}{\sqrt{\lambda f}} \sum_{\ell=1}^{N} H_{n}\left(y_{\ell}\right)\left[e^{i u(k) y_{\ell}}-(-1)^{n+1} e^{-i u(k) y} \ell\right] \\
* B\left(z_{\ell}\right) \exp \left[-\left(w_{m} y_{\ell} / 2\right)^{2}\right] .
\end{gathered}
$$

This expression contains all the details for the optical filter. It is useful to rewrite this expression to get it into a real-function form and to establish the form for the $g_{\ell}$.

First we define

$$
S 1(n, k, 2)=\frac{2 \sqrt{2} D O}{\sqrt{\lambda f}} A(n, k)(i)^{r(k)}
$$

using

$$
\begin{gathered}
W A\left(y_{\ell}\right)=\exp \left[-\left(w_{m} y_{\ell} / 2\right)^{2}\right], \\
y_{\ell}=x_{\ell}+z_{\ell}, \\
z_{\ell}=g_{\ell} / 2,
\end{gathered}
$$




$$
g_{\ell}=\left[\begin{array}{lll}
\alpha & 04 & h_{\ell}
\end{array}\right]_{q}
$$

Here the [ ]q represents the fact that the spot size adjustments are discrete rather than continuous. We note the constraints

$$
\begin{aligned}
& 0 \leqslant \alpha \leqslant 1 \\
& \left|h_{\ell}\right| \leqslant 1
\end{aligned}
$$

Because of the way a spot is generated we have certain conditions on the values of $\alpha$. For now, $\alpha$ is equal to 0.6 for the $x$-dimension pattern and to 0.7 for the $y$-dimension pattern. The discrete conditions are in steps of 1 for a range of \pm 30 units for the $x$ dimension and \pm 20 for the $y$ dimension. This latter condition means that the $y$ dimension has more quantization error. This fact can affect the final strategy for development of the optical filter. This point will be discussed in more detail in subsection 4.7. With the above definitions $A(n, k)$ becomes

$$
A(n, k)=\sum_{\ell=1}^{N} H_{n}\left(y_{\ell}\right) B\left(z_{\ell}\right) W A\left(y_{\ell}\right){ }_{\cos }^{\sin }\left[u(k) y_{\ell}\right] / D 0
$$

with

$$
\begin{aligned}
r(k) & =1 \text { for } k=1,3,5 \text { use } \sin \\
& =0 \text { for } k=2,4,6 \text { use cos. }
\end{aligned}
$$

and with

$$
W\left(y_{\ell}\right)=1 / W A\left(y_{\ell}\right) \cdot
$$

Notice that $A(n,-k)=-A(n, k)$ for $k$ odd and $A(n,-k)=A(n, k)$ for $k$ even. This means it is necessary to calculate only the positive $k$ values. We define four functions for convenience. Namely

$$
G 1(s, k 1, \ell) \equiv W\left(y_{\ell}\right) H_{s}\left(y_{\ell}\right) \cos \left[u(s) y_{\ell}\right] \sin \left[k 1 \Delta u y_{\ell}\right] \text {, }
$$




$$
\begin{aligned}
& \mathrm{G} 2(s, k 1, \ell) \equiv W\left(y_{\ell}\right) H_{s}\left(y_{\ell}\right) \cos \left[u(s) y_{\ell}\right] \cos \left[\begin{array}{lll}
k & \Delta u & y_{\ell}
\end{array}\right], \\
& G 3(s, k 1, \ell) \equiv W\left(y_{\ell}\right) H_{s}\left(y_{\ell}\right) \sin \left[u(s) y_{\ell}\right] \sin \left[\begin{array}{lll}
1 & \Delta u & y_{\ell}
\end{array}\right],
\end{aligned}
$$

and

$$
\mathrm{G} 4(\mathrm{~s}, \mathrm{kl}, \ell) \equiv W\left(\mathrm{y}_{\ell}\right) \mathrm{H}_{s}\left(\mathrm{y}_{\ell}\right) \sin \left[u(s) y_{\ell}\right] \cos \left[k 1 \Delta u y_{\ell}\right] \text {. }
$$

Using these definitions and noting the symmetry for $y_{\ell}$, we can further define in terms of some coefficients $B(s, k 1), C(s, k 1)$ the composite functions. For $s=1,3,5$

$$
U\left(s, y_{\ell}\right) \equiv \sum_{k 1=0}^{N_{2}}[B(s, k 1) G 4(s, k 1, \ell)+C(s, k 1) G 1(s, k 1, \ell)]
$$

and for $s=2,4,6$

$$
\begin{aligned}
& U\left(s, y_{\ell}\right) \equiv \sum_{k 1=0}^{N_{2}}[B(s, k 1) G 2(s, k 1, l)+C(s, k 1) G 3(s, k 1, \ell)] \\
& \left(\operatorname{note} N_{2} \leqslant 87\right) .
\end{aligned}
$$

The $h_{\ell}$ is finally defined as

$$
n_{\ell}=P \alpha \sum_{s=1}^{6} U\left(s, y_{\ell}\right)
$$

where the $P$ is adjusted to make contraint in eq (58) true.

A look at these equations shows that the relationships are nonlinear in $B(s, k 1)$ and $C(s, k 1)$. This means the process for determining the values of $B$ and $C$ requires an iteration process to finally get the proper values of $B$ and $C$. The sequence is to get the value of $A(n, k)$ to be as small as possible for the cases $n \neq k$ and to get the values of $A(n, n)$ to be approximately the same and as large as possible consistant with the constraints of the $q$.

The computation sequence is to start with a selected set of $B(s, k l)$ and $c(s, k l)$ and compute $g_{\ell}$ with choice of contraints in $\alpha$ and the $q$ operation in an interative manner until the values of $g_{\ell}$ are unchanged. For the range of 
values of $g_{\ell}$, this takes no more than three iterations. Once $g_{\ell}$ is determined, then it is possible to compute $A(n, s)$. The computation sequence described here is straightforward. The hard part is developing a means for improving the selected set of $B(s, k 1)$ and $C(s, k 1)$ to yet the best solution in as efficient a way as possible. The next subsection addresses this problem.

\subsection{Evaiuation of the Antisymmetric Quasilinear Part}

It is necessary to develop an iterative strategy to determine eventually the proper $B$ and $C$ values in some least squares sense. This subsection shows the necessary formulas and discusses the approximations and concepts necessary to get these formulas. In summary, the process is to get a quasilinear relationship between the changes in $B$ and $C$ and relate it to the difference between the current matrix $A(n, k)$ and the desired form for $A$. We now step through the formulation.

The change in $B$ is designated as $B 1(s, k 1)$. The change in $C$ is designated as $C 1(s, k 1)$. The equation relating the error to the desired form is written as

$$
-A^{\text {current }}(n, k)=\sum_{s=1}^{6} \sum_{k 1=1}^{87}\left[+\begin{array}{l}
A 1(n, k, s, k 1) B 1(s, k 1) \\
A 2(n, k, s, k 1) C 1(s, k 1)
\end{array}\right]
$$

Here, $A^{\text {current }}(n, k)$ corresponds to the terms computed in ey (59) after the $g_{\ell}$ terms have been stabilized to self consistancy.

The new $B$ and new $C$ are obtained from the old $B$ and old $C$ by

$$
\begin{aligned}
& B^{N}(s, k 1)=B^{0}(s, k 1)+B 1(s, k 1) \\
& C^{N}(s, k 1)=C^{0}(s, k 1)+C 1(s, k 1)
\end{aligned}
$$

with the constraint that the $\mathrm{k}_{\ell}=0$ case has no $\mathrm{B} 1$ and $\mathrm{Cl}$ terms. Instead $\mathrm{B}$ and $C$ here are restricted to

$$
\begin{array}{ll}
B(s, 0)=1 & 1 \leqslant s \leqslant 6 \\
C(s, 0)=0 &
\end{array}
$$


This constraint guarantees that the computer search for the ideal solution will not cause the $B$ and $C$ to be zero.

If we replace $y_{\ell}$ by $x_{\ell}$ and drops the $\left(z_{\ell}\right)^{3}$ term in $B\left(z_{\ell}\right)$, we get a term linear in $B(s, k 1)$ and $C(s, k 1)$. As long as the primary source of mixing between the different modes is dominated by the linear term we have a good approximation. Since this is so, we can find the $A 1$ and $A 2$ matrices. If necessary, we could use the old $g_{\ell}$ and replace $x_{\ell}$ by $y_{\ell}$ to get the yuasilinear form for the $\mathrm{A} 1$ and $\mathrm{A} 2$ matrices. In this case, the computer analysis would involve another level of computation. Fortunately, it is not necessary to do this complex process. We now make a number of definitions. First, we have the Fourier transform of the cross-correlation product written as

$$
\begin{aligned}
& Q 1[n, s, k x]=\sum_{\ell=1}^{N} H_{n}\left(x_{\ell}\right) H_{s}\left(x_{\ell}\right) \cos \left(k x \cdot \Delta u \cdot x_{\ell}\right) \\
& Q 1[n, s, k x]=\sum_{\ell=1}^{N} H_{n}\left(x_{\ell}\right) H_{s}\left(x_{\ell}\right) \cos \left(k x \cdot \Delta u \cdot x_{\ell}\right)
\end{aligned}
$$

Next, we note the symmetries as

$$
\begin{aligned}
& Q 1[n, s,-k x]=Q 1[n, s, k x] \\
& Q 2[n, s,-k x]=-Q 2[n, s, k x]
\end{aligned}
$$

Also, there are four spatial frequency terms to be defined, namely

$$
\begin{aligned}
& k a \cdot \Delta u \equiv k 1 \cdot \Delta u+u(k)+u(s) \\
& k b \cdot \Delta u \equiv-k 1 \cdot \Delta u+u(k)+u(s) \\
& k c \cdot \Delta u \equiv k 1 \cdot \Delta u+u(k)-u(s) \\
& k d \cdot \Delta u \equiv-k 1 \cdot \Delta u+u(k)-u(s) .
\end{aligned}
$$

Finally, a projection operator for $k$ and $s$ can be defined as 


$$
P_{ \pm}(k) \equiv \frac{1}{2}\left(1 \pm(-1)^{k}\right) \text {. }
$$

Using these definitions, we can write out the form of $A 1$ and $A 2$ where the top siyn corresponds to the $A 1$ case and the bottom sign corresponds to the A2 case.

$$
\begin{aligned}
& A 12(n, k, s, k 1)=\frac{P \alpha}{8} \quad\left[Q 1(n, s, k a)\left[-P_{+}(k) P_{+}(s) \pm P_{-}(k) P_{-}(s)\right]\right] \\
& {\left[Q 1(n, s, k b)\left[F_{+}(k) P_{+}(s)+P_{-}(k) P_{-}(s)\right]\right.} \\
& {\left[Q 1(n, s, k c)\left[ \pm P_{+}(k) P_{+}(s)+P_{-}(k) P_{-}(s)\right]\right.} \\
& {\left[Q 1(n, s, k d)\left[P_{+}(k) P_{+}(s) \pm P_{-}(k) P_{-}(s)\right]\right.} \\
& {\left[Q 2(n, s, k a)\left[ \pm P_{+}(k) P_{-}(s)+P_{-}(k) P_{+}(s)\right]\right.} \\
& {\left[Q 2(n, s, k b)\left[P_{+}(k) P_{-}(s) \mp P_{-}(k) P_{+}(s)\right]\right.} \\
& {\left[Q 2(n, s, k c)\left[P_{+}(k) P_{-}(s) \mp P_{-}(k) P_{+}(s)\right]\right.} \\
& {\left[Q 2(n, s, k d)\left[ \pm P_{+}(k) P_{-}(s)-P_{-}(k) P_{+}(s)\right]\right.}
\end{aligned}
$$

We are finally able to set up the proper numerical analysis problem. First we yroup the $n, k$ labels into a single label and we group the $s, k 1$, with $\mathrm{B} 1$ or $\mathrm{Cl}$ into a single label, thus

$$
\begin{aligned}
& \text { Map } n \neq k \text { into } 1 \leqslant k 3 \leqslant 30 \\
& \text { Map } \mathrm{s}, \mathrm{K} 1, \mathrm{~B}, \mathrm{C} \text { into } 1 \leqslant \mathrm{~K} 4 \leqslant 1044 .
\end{aligned}
$$

We next construct a rectangular matrix $M$ and two vectors $T$ and $E$ as follows

$$
\begin{aligned}
& M[K 4, K 3]=\left[\begin{array}{l}
A 1 \otimes A 2
\end{array}\right], \\
& T(K 4)=\left[\begin{array}{lll}
B 1 & \oplus & C 1
\end{array}\right],
\end{aligned}
$$


and

$$
E(K 3)=-A^{\text {current }}(n, k) n \neq k \text {. }
$$

It is now possible to write eq (66) in matrix form as

$$
T M=E \text {. }
$$

We now do a singular value decomposition of $M$ into two orthonormal matrices $U$ and $V$ and into a diagonal matrix $D$ [19]. Thus,

$$
M=U D V^{\top} \text {. }
$$

The solution in a least squares sense is given by

$$
T=E V D 1 U^{\top}
$$

Here the requirement is made that DI is inverted in a constrained way. Thus,

$$
D 1=1 / D \text { for } D\left(\ell_{1}\right) \geqslant 10^{-3} D_{\operatorname{riax}}
$$

or

$$
=0 \text { for } D\left(\ell_{1}\right)<10^{-3} D_{\text {max }} \text {, }
$$

where

$$
1 \leqslant \ell_{1} \leqslant 30 .
$$

We select this level of cutoff because the analysis is carried to the 0.1 percent level. To do better would require consideration of the dc and error terms. We suggest that it is premature to work to a higher accuracy than this 0.1 percent.

There remains only one step in the determination of the $B$ and $C$ matrices. It is necessary to determine when the error in the cross-correlation process is sufficiently small. We define the error as

$$
\text { ERROR }=\sum_{n \neq k}|A(n, k)| / 30 .
$$


Define the signal as

$$
\text { SIGNAL }=\sum_{n=k}|A(n, k)| / 6
$$

Finally, accept the computer results as correct when

$$
\text { ERROR } \leqslant 10^{-3} \text { SIGNAL }
$$

4.7 Two Fabrication Strategies--Which One Is Better?

We define briefly how the filter is constructed. The sequence starts with the construction of a spot. A spot has up to 40 lines written in the $y$ dimension, and each of these lines has a length ranging from 2 to 8 um in length. The construction sequence is to generate a cell of 20 by 20 spots and then to go to the next cell along the $x$ dimension for a total of 350 cells. The of cells are generated in the y direction one row at a time until there are 350 such rows of cells.

There are two basic construction strategies: The first is to have every spot written with all 40 lines and to vary the length of the lines according to the $g_{\ell}$ pattern given by the theory in this paper with $\alpha=0.6$. This pattern is generated for every spot in a cell and for every row of cells. The resulting pattern would then look like a one-dimensional pattern with variable width lines in the $x$ dimension and running in the $y$ dimension the full $70 \mathrm{~mm}$. This master plate is then copied by contact printing to generate the secondary plates. Two secondary plates are put together with one plate rotated $90^{\circ}$ relative to the other and placed so that the two light absorbing parts of each plate are next to each other. In this way we have constructed a twodimensional pattern with maximum accuracy relative to the q operation. The problems are to make sure the optical axis of each plate is exactly the same and that the rotation angle is truly $90^{\circ}$.

The second strategy is to generate the $y$ pattern as well as the $x$ pattern on a single master plate. The secondary plates are then generated from this master plate by contact printing. Here the errors of positioning the plates 
are eliminated. The trade-off is a reduced accuracy in the q operation along the $y$ dimension.

Both techniques need to be tested to determine which is more appropriate. At this time, we think the first strategy will be the more accurate as well as more flexible. The key question will be how well the transfer process works to create secondary plates. If it works well, then the cost of fabrication of plates will be substantually reduced. If it does not work well, then it may be necessary to generate each secondary plate just as the master was done. This path will be expensive.

4.8 Necessary Work to Realize the Apparatus

The theoretical work has been completed and summarized in this paper. The computer codes are written and are believed to be debugged. What needs to be done now are:

1. Construction of the optical filter via the two strategies and testing those units to confirm that there are no bugs in the computer code and to check the accuracy of construction of the optical filter.

2. Construction of the tapered fiber with the specifications indicated in this paper.

3. Construction of the 3-by-3 tapered fiber units with the piezoelectric material. Testing of these units to verify they work as intended. Issues that will need to be addressed are the time stability of the units and hysteresis effects of the piezoelectric material.

4. Construction of the two optics, the telescopes, and Fourier transform pair of lens, of this apparatus and testing them.

5. Finally the complete construction of the electric field apparatus and its testing for accuracy and stability.

5. Conclusion

We now summarize this paper. There is the theoretical analysis of an electric field measuring apparatus. The key mathematical formulas for each stage are shown and a concrete set of values are selected so that you can have a picture of the apparatus. 
The paper was structured to show the basic ideas and then to show the complex ideas for construction of the optical filter.

In the basic ideas section, you saw first that there is need for a pair of telescopes to match the beamwidth and phase front of the incident laser beam to that needed at the optical filter. Second, you saw how the optical filter is constructed and how its effect on the laser beam is transformed by a pair of lenses. Third, you learned that there are selected locations where there are cross-correlation structures which contain information on the strength, phase, and polarization of the modes used to represent the transverse spatial features of the electric field. Fourth, you learned that the key step in measuring the electric field requires an array of tapered optical fibers to capture the fundamental cross-correlation mode and to make it the fundamental propagation mode of the fibers. You learned how this capture can be performed and were given estimates of the errors involved in this capture. Finally, you were shown how the beams from the fibers were separated according to polarization and how the detectors were set up to measure the resulting beams. At this point, you have the potential of 72 time series signals which characterized the strength of each orthonormal mode in the original electric field. In this paper, we assumed that the power is measured for each mode.

In the construction of the optical filter section, you saw the mathematics necessary for its construction and estimates of the errors involved in making approximations to these mathematics. You learned that the basic construction of the optical filter requires solution to nonlinear equations and some very complex mathematics.

We finish this conclusion with two comments:

1. Once the apparatus is constructed, it will be possible to do a calibration process with a single well characterized $\mathrm{cw}$ beam to evaluate the unit and to use some form of matrix analysis to reduce substantially some of the remaining errors. This calibration process will define precisely along the optical axis where we have made the electric field measurement. Other locations would have their electric fields defined by using either a new calibration scan or by using the theory to propagate the laser beam to the new locations. 
2. We have emphasized Hermite modes in this discussion. In order to have good separation between the cross-correlation structures in the Fourier plane, it is necessary to stay away from sharp cut-off structures for representing the orthonormal set. For example, a plane wave illuminating the optical filter would imply an Airy pattern where the effective range of the electric field is 44 times greater than that implied by Hermite modes. This means that beam steering of the cross-correlation process would have to be incorporated to account for this kind of smearing of the cross-correlation structure. We suggest it is best to avoid this problem by using carefully smoothed corners in the orthonormal mode set so that this ringing in the Fourier plane is minimal [22].

In a complex work of this sort there are a larye number of people and groups who have helped me at various stages in the work. Matt Young, Aaron Sanders, Don Jennings, Norris Nahman, Bruce Danielson, Douglas Franzen, Gordon Day, Alvin Rasmussen, Robert Gallawa all helped in giving me their technical advice and thoughts. The members of the Scientific Computing Division, namely John Gary, Roland Sweet, Michael Maish, James Williams, Lloyd Fosdick, Robert Schnabel, and David Lovering have all helped by giving me their thoughts on the mathematics and by making sure the computer would work for me. Gary and Schnabel were especially helpful in developing the appropriate Singular Value Decomposition Strategies. The excellent drafting was done by Nick Sanchez. The manuscript preparation was handled by Edie DeWeese and secretarial activities were handled by Susie Rivera. The Combined Calibration Group (CCG) of the Department of Defense has partially funded the background necessary to get this work where it is today.

\section{References}

[1] Johnson, Eric G. Jr. Laser beam profile measurements using spatial sampling, Fourier optics, and holography. Nat. Bur. Stand. (U.S.) Tech. Note 1009; 1979 January. 
[2] Johnson, Eric G. Jr. Design of a reflection apparatus for laser beam profile measurements Nat. Bur. Stand. (U.S.) Tech. Note 1015; 1979 July.

[3] Johnson, Éric G. Jr. Beam-profile measurements of laser pulses using a spatial filter to sample the Hermite modes for a string of pulses. Nat. Bur. Stand. (U.S.) Tech. Note 1057; 1982 September.

[4] Johnson, Eric G. Jr. Using optical processing to find the beam profile of a laser pulse (theory). Proc. SPIE 499:75-88; 1985.

[5] Kogelnik, H.; Li, T. Laser beams and resonators. Proc. IEEE, 54:1312$1329 ; 1966$ October.

[6] Cathey, W. Thomas. Optical information processing and holography. John W. Wiley \& Sons, pp. 1-200; 1974.

[7] Allan, W. R. Fiber optics, theory and practice. Plenum Press, Chapter 9, pp. 177-197; 1973

[8] Lindgren, L.; Vilhelmsson, K. Mode excitation in graded-index optical fibers. J. Lightwave Tech. LT-2(2):559-563; 1984 August.

[9] Ozeki, Takeshi; Ito, Takao; Tamura, Toshifumi. Tapered section of multimode cladded fibers as mode filter and mode analyzers. Appl. Phys. Lett. 26(7):386-388; 1975.

[10] Montalti, F.; Val1i, M.; Vannucci, R. Optical fiber technology-analysis of tapered graded-index fibers. Elect. Commun. 56(4):431-434; 1981.

[11] Modern examples of piezoelectric coefficients are shown in report PD-9247 Modern Piezoelectric Ceramics, Vernitron Corporation. No endorsement is implied by using this example.

[12] Vidosic, J. P. Mechanics of material. Chapt. 5 in Standard handbook for mechanical engineers, 8th Ed. T. Baumeister, E. A. Avalline, and T. Baumeister III, eds. Macgraw Hi11; 1978. pp. 5-38

[13] Hecht, Eugene; Zajac, Alfred. Optics. Addison-Wesley; 1979. p. 234.

[14] Barnoski, M. K. Introduction to integrated optics. Plenum; 1974.

[15] Teich, M. C. Infrared heterodyne detection. Proc. IEEE 56:37-46; 1968.

[16] Sze, S. M. Physics of semiconductor devices. Wiley-Interscience, pp. 625-686; 1969.

[17] Day, G. W.; Hamilton, C. A.; Gruzensky, P. M.; Phelan, R. J., Jr. Performance and characteristics of polyvinylidene fluoride pyroelectric detectors. Ferroelect. 10:99-102; 1976. 
[18] National Computer Holography Facility at Aerodyne Research, Inc., see discussion in Laser Focus; November 1982 on pages 42-46. The design specifications where chosen since they have been well specified for use by the general public.

[19] Nash, J. C. Compact numerical methods for computers: linear algebra and function minimisation. John Wiley \& Sons; 1979. Of particular interest here is the discussion in chapter 3 on the singular value decomposition procedure.

[20] Wilson, Scott D.; Reed, Timothy. Beam and spot profiles measurement methods for optical storage systems. SPIE 499:65-74; 1984.

[21] Frank, J. D. Beam profile measurement for target designators. SPIE 499: $56-64 ; 1984$.

[22] Priestley, M. B. Probability and mathematical statistics. Spectral Analysis and Time Series, Vol. 1, Univariate Series, Academic Press; 1981. Here are some examples of cutoff techniques to avoid ringing. 
NBS-114A (REV. 2-8C)

U.S. DEPT. OF COMM.

BIBLIOGRAPHIC DATA

SHEET (See instructions)

1. PUBLICATION OR

REPORT NO.

NBS TN-1084

August 1985

4. TITLE AND SUBTITLE

Direct Measurement of the Electric Field of a Laser Pulse--Theory

5. $\operatorname{AUTHOR}(\mathrm{S})$

Eric G. Johnson, Jr.

6. PERFORMING ORGANIZATION (If joint or other than NBS, see instructions)

NATIONAL BUREAU OF STANDARDS

DEPARTMENT OF COMMERCE

WASHINGTON, D.C. 20234

9. SPONSORING ORGANIZATION NAME AND COMPLETE ADDRESS (Street, City, Stote, ZIP)

7. Contract/Grant No.

8. Type of Report \& Period Covered

10. SUPPLEMENTARY NOTES

Document describes a computer program; SF-185, FIPS Software Summary, is attached.

11. ABSTRACT (A 200-word or less factual summary of most significant information. If document includes a significant bibliography or literature survey. mention it here)

We consider realizing an electric field measuring apparatus by using optical processing, tapered optical fibers, and a pair of detectors at the end of each optical fiber. Using an appropriate computer-generated optical filter, we show it is possible to discriminate among a set of orthonormal modes used to represent the spatial features of the electric field with a signal-to-noise ratio of at least 100 to 1 . If the positioning accuracies for various parts of the apparatus are properly set up, it is expected that the signal-to-noise ratio could be about 1000. The purpose of the tapered and graded-index fiber is to select the fundamental propagating mode in a fiber and to attenuate the other modes. The existence of this fiber allows the precise determination of the strength of each of the orthonormal modes being used as the spatial basis of the electric field before the optical processing. The detectors then measure the strength of each fundamental mode. There are 36 such modes for the apparatus under discussion--six for the $x$ dimension and six for the $y$ dimension. Propagation of the laser pulse is assumed to be along the $z$ axis. We use two detectors for each mode so that the polarization is determined.

This paper presents the conflicts in the design and gives a solution. The complete evaluation requires assembly of the proposed apparatus to assess final accuracy.

12. KEY WORDS (Six to twelve entries; alphabetical order; capitalize only proper names; and separate key words by semicolons) beam profile measurements; cross-correlation; electric field measurement; graded-index fibers; laser pulses; matched filters; mode analysis; mode filtering; numerical analysis; optical fibers; optical filter; singular value decomposition; total beam control

13. AVAILABILITY

X] Unlimited

$\square$ For Official Distribution. Do Not Release to NTIS

[X] Order From Superintendent of Documents, U.S. Government Printing Office, Washington, D.C. 20402.

14. NO. OF

PRINTED PAGES

[ Order From National Technical Information Service (NTIS), Springfield, VA. 2216I

60

15. Price 




\section{Technical Publications}

\section{Periodicals}

Journal of Research-The Journal of Research of the National Bureau of Standards reports NBS research and development in those disciplines of the physical and engineering sciences in which the Bureau is active. These include physics, chemistry, engineering, mathematics, and computer sciences. Papers cover a broad range of subjects, with major emphasis on measurement methodology and the basic technology underlying standardization. Also included from time to time are survey articles on topics closely related to the Bureau's technical and scientific programs. As a special service to subscribers each issue contains complete citations to all recent Bureau publications in both NBS and non-NBS media. Issued six times a year.

\section{Nonperiodicals}

Monographs-Major contributions to the technical literature on various subjects related to the Bureau's scientific and technical activities.

Handbooks-Recommended codes of engineering and industrial practice (including safety codes) developed in cooperation with interested industries, professional organizations, and regulatory bodies.

Special Publications-Include proceedings of conferences sponsored by NBS, NBS annual reports, and other special publications appropriate to this grouping such as wall charts, pocket cards, and bibliographies.

Applied Mathematics Series-Mathematical tables, manuals, and studies of special interest to physicists, engineers, chemists, biologists, mathematicians, computer programmers, and others engaged in scientific and technical work.

National Standard Reference Data Series-Provides quantitative data on the physical and chemical properties of materials, compiled from the world's literature and critically evaluated. Developed under a worldwide program coordinated by NBS under the authority of the National Standard Data Act (Public Law 90-396).

NOTE: The Journal of Physical and Chemical Reference Data (JPCRD) is published quarterly for NBS by the American Chemical Society (ACS) and the American Institute of Physics (AIP). Subscriptions, reprints, and supplements are available from ACS, 1155 Sixteenth St., NW, Washington, DC 20056.

Building Science Series-Disseminates technical information developed at the Bureau on building materials, components, systems, and whole structures. The series presents research results, test methods, and performance criteria related to the structural and environmental functions and the durability and safety characteristics of building elements and systems.

Technical Notes-Studies or reports which are complete in themselves but restrictive in their treatment of a subject. Analogous to monographs but not so comprehensive in scope or definitive in treatment of the subject area. Often serve as a vehicle for final reports of work performed at NBS under the sponsorship of other government agencies.

Voluntary Product Standards-Developed under procedures published by the Department of Commerce in Part 10, Title 15, of the Code of Federal Regulations. The standards establish nationally recognized requirements for products, and provide all concerned interests with a basis for common understanding of the characteristics of the products. NBS administers this program as a supplement to the activities of the private sector standardizing organizations.

Consumer Information Series-Practical information, based on NBS research and experience, covering areas of interest to the consumer. Easily understandable language and illustrations provide useful background knowledge for shopping in today's technological marketplace.

Order the above NBS publications from: Superintendent of Documents, Government Printing Office, Washington, DC 20402.

Order the following NBS publications-FIPS and NBSIR's-from the National Technical Information Service, Springfield, VA 22161.

Federal Information Processing Standards Publications (FIPS PUB)-Publications in this series collectively constitute the Federal Information Processing Standards Register. The Register serves as the official source of information in the Federal Government regarding standards issued by NBS pursuant to the Federal Property and Administrative Services Act of 1949 as amended, Public Law 89-306 (79 Stat. 1127), and as implemented by Executive Order 11717 (38 FR 12315, dated May 11, 1973) and Part 6 of Title 15 CFR (Code of Federal Regulations).

NBS Interagency Reports (NBSIR)-A special series of interim or final reports on work performed by NBS for outside sponsors (both government and non-government). In general, initial distribution is handled by the sponsor; public distribution is by the National Technical Information Service, Springfield, VA 22161, in paper copy or microfiche form. 
U.S. Department of Commerce

National Bureau of Standards

Gaithersburg, MD 20899

Official Business

Penalty for Private Use $\$ 300$

POSTAGE AND FEES PAID U.S. DEPARTMENT OF COMMERCE COM-215

SPECIAL FOURTH-CLASS RATE BOOK 